\title{
Role of Anticausal Inverses in Multirate Filter-Banks-Part II: The FIR Case, Factorizations, and Biorthogonal Lapped Transforms
}

\author{
P. P. Vaidyanathan, Fellow, IEEE, and Tsuhan Chen, Member, IEEE
}

\begin{abstract}
In a companion paper, we studied the systemtheoretic properties of discrete time transfer matrices in the context of inversion, and classified them according to the types of inverses they had. In particular, we outlined the role of CAusal FIR matrices with AntiCAusal FIR inverses (abbreviated cafacafi) in the characterization of FIR perfect reconstruction (PR) filter banks. Essentially all FIR PR filter banks can be characterized by causal FIR polyphase matrices having anticausal FIR inverses. In this paper, we introduce the most general degree-one cafacafi building block, and consider the problem of factorizing cafacafi systems into these building blocks. Factorizability conditions are developed. A special class of cafacafi systems called the biorthogonal lapped transform (BOLT) is developed, and shown to be factorizable. This is a generalization of the well-known lapped orthogonal transform (LOT). Examples of unfactorizable cafacafi systems are also demonstrated. Finally it is shown that any causal FIR matrix .with FIR inverse can be written as a product of a factorizable cafacafi system and a unimodular matrix.
\end{abstract}

\section{INTRODUCTION}

$\mathbf{I}^{\prime}$ $\mathrm{N}$ A companion paper [1], we studied the system-theoretic properties of discrete time transfer matrices in the context of inversion, and classified them according to the types of inverses they had. In particular, we outlined the role of CAusal FIR matrices with AntiCAusal FIR inverses (cafacafi) in the characterization of FIR perfect reconstruction filter banks.

Briefly, Fig. 1(a) represents a maximally decimated filter bank with identical decimation ratios in all the channels. This can be redrawn in polyphase form as in Fig. 1(b). The system has the perfect reconstruction property (i.e., $\hat{x}(n)=x(n)$ in absence of subband quantizers) if and only if $\mathbf{R}(z)=\mathbf{E}^{-1}(z)$. See [1] for detailed references on this topic. An FIR filter bank is one where $\mathbf{E}(z)$ and $\mathbf{R}(z)$ are FIR. In [1] we argued that in the FIR case, if we study the cafacafi class of matrices $\mathbf{E}(z)$, it is sufficient to characterize practically all FIR PR filter banks.

In contrast, the family of causal FIR transfer matrices with causal FIR inverses (i.e., unimodular matrices in $z^{-1}$ ) are not very useful in characterizing the class of all FIR PR filter

Manuscript received October 29, 1993; revised November 1, 1994. This work was supported by the Office of Naval Research under Grant NO001493-1-023I, Tektronix, Inc., and Rockwell, International. The associate editor coordinating the review of this paper and approving it for publication was Dr. Truong Nguyen.

P.P. Vaidyanathan is with the Department of Electrical Engineering, California Institute of Technology, Pasadena, CA 91125 USA.

T. Chen was with the Department of Electrical Engineering, California Institute of Technology, Pasadena, CA 91125 USA. He is now with AT\&T Laboratories, Holmdel, NJ 07733 USA.

IEEE Log Number 9410287.

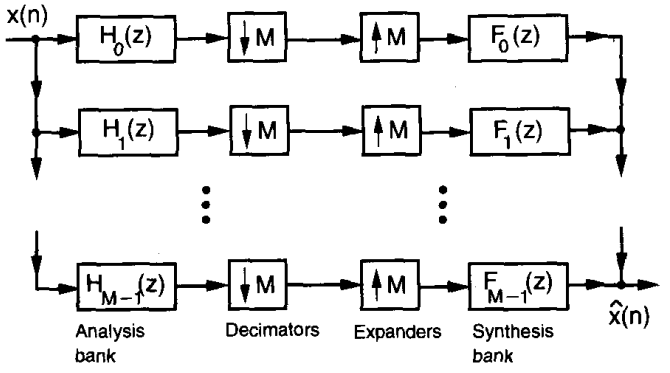

(a)

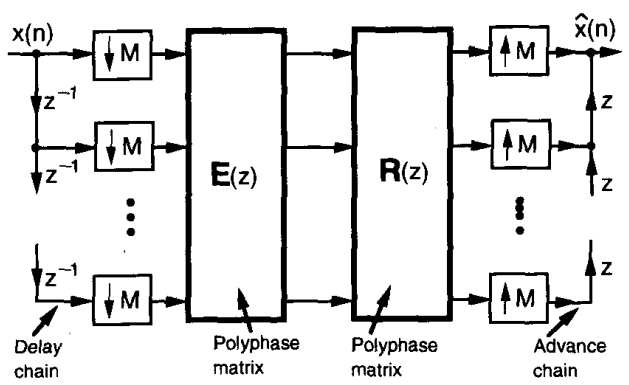

(b).

Fig. 1. (a) Maximally decimated filter bank; (b) polyphase representation

banks. First, restricting the polyphase matrix to be unimodular results in a loss of generality; given a causal FIR system with arbitrary FIR inverse, we cannot in general multiply it with a delay $z^{-I}$ to obtain a causal FIR system with a causal FIR inverse. Furthermore, as we will see at the end of Section IIA, unimodular matrices cannot in general be factorized into degree-one unimodular building blocks. ${ }^{1}$ For these reasons, we will not pursue the possibility of characterizing FIR PR systems in terms of unimodular matrices alone. The class of cafacafi systems are more useful than unimodular systems for this purpose.

In this paper we will use the results of [1] to obtain certain fundamental FIR building blocks with FIR inverses. These building blocks can be considered to be the biorthogonal versions of the orthonormal (paraunitary) systems reported

'Even though it is well-known [2] that unimodular matrices can be expressed as products of three kinds of elementary matrices, that would not be a useful parameterization for filter bank design. For it would not yield us a structure with a fixed number of multipliers which can be optimized to design the filter responses. 
earlier [3]-[5]. We will consider the factorization of cafacafi systems using these building blocks and develop some results in this direction. For convenience, we state from [1] two of the system-theoretic results that play a crucial role in this paper:

1) An $M \times M$ causal LTI system $\mathbf{G}(z)$ has an anticausal inverse if and only if the realization matrix

$$
\mathcal{R} \triangleq\left[\begin{array}{ll}
\mathbf{A} & \mathbf{B} \\
\mathbf{C} & \mathbf{D}
\end{array}\right]
$$

of any minimal realization of $\mathbf{G}(z)$ is nonsingular (see Theorem 5.1 of [1]). Whether the anticausal inverse is FIR or not is not addressed by this result.

2) Let $\mathbf{G}(z)$ be an $M \times M$ causal FIR system with FIR inverse. Then, the inverse is anticausal FIR if and only if $[\operatorname{det} \mathbf{G}(z)]=c z^{-N}$, where $N=$ McMillan degree of $\mathbf{G}(z)$ (see Theorem 5.3 of [1]).

\section{A. Paper Outline}

Based on the results of [1], we will establish a number of positive and negative results pertaining to factorization of FIR systems with FIR inverses. The road map is as follows:

1) In Section II, we present a degree-one FIR building block, and establish conditions under which it will have different types of FIR inverses (causal, anticausal, and so forth). The unimodular building block (i.e., the one with causal FIR inverse) which results from this study is used to demonstrate that an arbitrary unimodular matrix may not be factorizable into degree one unimodular blocks.

2) The degree-one cafacafi building block which results from our discussion will be used in Section II-B to derive conditions under which arbitrary cafacafi systems can be factorized into these building blocks. Even though the building block is the most general degree-one cafacafi system (as we show later in Section IV), we will see in Section VI that it cannot be used to factorize arbitrary cafacafi systems.

3) In Section III, we restate the factorizability conditions for cafacafi systems in terms of state space parameters.

4) Using this, we show in Section IV that a subclass of matrices called the biorthogonal lapped transforms (BOLT), which is a generalization of the lapped orthogonal transform LOT [6]-[8], can always be factorized into degree one cafacafi building blocks.

5) In Section V, we study FIR transfer matrices of the form $I-\mathcal{U} \mathcal{V}^{\dagger}+z^{-1} \mathcal{U} \mathcal{V}^{\dagger}$ and show that many properties of the inverse can be deduced from the eigenvalues of $\mathcal{V}^{\dagger} \mathcal{U}$ (Theorem 5.2). We use this to find necessary and sufficient conditions for any first order FIR matrix to be a BOLT. In particular, we impose conditions on the degree-one factors derived in Section II, guaranteeing the BOLT property structurally.

6) In Section VI, we derive examples of cafacafi systems that cannot be factorized into degree one building blocks, and introduce degree two building blocks. It is also shown that there exist cafacafi systems which cannot be factorized using any combination of these building blocks.

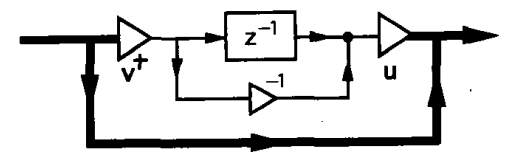

Fig. 2. Implementation of the degree-one building block. The thick lines indicate vector signals and thin lines (such as the input and output of the delay element) indicate, scalar signals.

7) However, in Section VII we show that any causal FIR matrix with FIR inverse can be written as a product of a factorizable cafacafi system and a unimodular matrix. This factorization, however, is not minimal as we shall explain.

All notations and acronyms will be exactly as in [1].

\section{SYNTHEsIS UsING DEgREe-ONE BUILDING BlockS}

In this section, we introduce the general degree-one causal FIR building block of the form

$$
\mathbf{V}(z)=\mathbf{I}-\mathbf{u v}^{\dagger}+z^{-1} \mathbf{u} \mathbf{v}^{\dagger}
$$

where $\mathbf{u}$ and $\mathbf{v}$ are $M \times 1$ vectors, and study its properties. In particular, its role in the synthesis of FIR causal systems with anticausal FIR inverses will be studied. Because of the appearance of the outer product $\mathbf{u v}^{\dagger}$, the building block is said to be diadic-based. Fig. 2 shows a structure for this system. Note that $\mathbf{V}(1)=\mathbf{I}$

\section{A. Properties of the Degree-One Building Block}

Theorem 2.1: Consider the $M \times M$ system $\mathbf{V}(z)=\mathbf{I}-$ $\mathbf{u v}^{\dagger}+z^{-1} \mathbf{u v} \mathbf{v}^{\dagger}$, where $\mathbf{u}$ and $\mathbf{v}$ are $M \times 1$ vectors (so that the degree $=1$ unless $\mathbf{u}$ or $\mathbf{v}$ is zero). Then, the following are true:

1) $[\operatorname{det} \mathbf{V}(z)]=1+\mathbf{v}^{\dagger} \mathbf{u}\left(z^{-1}-1\right)$

2) Let $\mathbf{v}^{\dagger} \mathbf{u}=1$, so that $[\operatorname{det} \mathbf{V}(z)]=z^{-1}$. In this case, $\mathbf{V}^{-1}(z)=\mathbf{V}\left(z^{-1}\right)=\mathbf{I}-\mathbf{u} \mathbf{v}^{\dagger}+z \mathbf{u} \mathbf{v}^{\dagger}$. That is, the inverse is anticausal FIR. If $\mathbf{u}=\mathbf{v}$, then $\mathbf{V}(z)$ becomes the paraunitary building block known before [5].

3) Let $\mathbf{v}^{\dagger} \mathbf{u}=0$, so that $[\operatorname{det} \mathbf{V}(z)]=1$ (i.e., $\mathbf{V}(z)$ is unimodular in $\left.z^{-1}\right)$. In this case, $\mathbf{V}^{-1}(z)=\mathbf{I}+\mathbf{u} \mathbf{v}^{\dagger}$ $z^{-1} \mathbf{u v} \mathbf{v}^{\dagger}$ which is causal FIR.

Proof: Let $\mathbf{x}_{i}, 0 \leq i \leq M-2$ be vectors orthogonal to v. Then, $\mathbf{V}(z) \mathbf{x}_{i}=\mathbf{x}_{i}$ so that there are $M-1$ eigenvectors with eigenvalue unity. Next, by substitution we see that $\mathbf{V}(z) \mathbf{u}=\left(1+\mathbf{v}^{\dagger} \mathbf{u}\left(z^{-1}-1\right)\right) \mathbf{u}$ so that $\left(1+\mathbf{v}^{\dagger} \mathbf{u}\left(z^{-1}-1\right)\right)$ is an eigenvalue. When $\mathbf{v}^{\dagger} \mathbf{u} \neq 0, \mathbf{u}$ is not in the span of $\left\{\mathbf{x}_{i}\right\}$. Therefore, we have found $M$ independent eigenvectors including $\mathbf{u}$, and all but one have eigenvalue equal to unity Thus

$$
\operatorname{det} \mathbf{V}(z)=1+\mathbf{v}^{\dagger} \mathbf{u}\left(z^{-1}-1\right)
$$

When $\mathbf{v}^{\dagger} \mathbf{u}=0$, it can be shown that there are no eigenvectors of $\mathbf{V}(z)$ other than the $\mathbf{x}_{i}$ (or their linear combinations). For this note that $\mathbf{V}(z) \mathbf{w}=\mathbf{w}+\left(\mathbf{v}^{\dagger} \mathbf{w}\right)\left(z^{-1}-1\right) \mathbf{u}$ for any $\mathbf{w}$. If $\mathbf{w}$ is an eigenvector, then either $\mathbf{i}) \mathbf{w}$ is aligned to $\mathbf{u}$ or ii) 
$\mathbf{v}^{\dagger} \mathbf{w}=0$. Since $\mathbf{v}^{\dagger} \mathbf{u}=0$, condition i) implies $\mathbf{v}^{\dagger} \mathbf{w}=0$ which is condition ii) again. The condition $\mathbf{v}^{\dagger} \mathbf{w}=0$ means, of course, that $\mathbf{w}$ is a linear combination of $\mathbf{x}_{i}$ 's. Therefore, all the eigenvectors are in the span of $\mathbf{x}_{i}$ 's, and the common eigenvalue is unity. Thus, $[\operatorname{det} V(z)]=1$, that is, (3) holds even with $\mathbf{v}^{\dagger} \mathbf{u}=0$.

The stated forms of the inverses in parts 2 and 3 can be verified by direct multiplication of $\mathbf{V}(z)$ with the claimed inverse and using $\mathbf{u}^{\dagger} \mathbf{v}=0$ or 1 as the case may be.

Comments:

1) For $\mathbf{v}^{\dagger} \mathbf{u}=1$, the following identity is easily verified:

$$
\mathbf{I}-\mathbf{u v}^{\dagger}+z^{-K} \mathbf{u v} \mathbf{v}^{\dagger}=\prod_{u c k \text { times }}\left(\mathbf{I}-\mathbf{u} \mathbf{v}^{\dagger}+z^{-1} \mathbf{u v} \mathbf{v}^{\dagger}\right)
$$

2) Smith-McMillan forms (reviewed in Section IV-A of [1]). Since $\mathbf{v}^{\dagger} \mathbf{u}=1$ implies that $\mathbf{V}(z)$ has an anticausal FIR inverse; therefore, by Theorem 5.2 of [1], the Smith-McMillan form of $\mathbf{V}(z)$ is $\left[\begin{array}{cc}z^{-1} & 0 \\ 0 & I\end{array}\right]$. On the other hand, $\mathbf{v}^{\dagger} \mathbf{u}=0$ implies that $\mathbf{V}(z)$ is unimodular in $z^{-1}$, and the Smith-McMillan form of $\mathbf{V}(z)$ is the identity matrix modified as follows: the first diagonal element is replaced with $z^{-1}$ and the last diagonal element replaced with $z$.

3) Let $\mathbf{u v}^{\dagger} \neq 0$ to avoid trivialities. Then we can show the following: $\mathbf{V}(z)$ has an i) anticausal inverse if and only if $\mathbf{v}^{\dagger} \mathbf{u} \neq 0$, ii) FIR inverse if and only if $\mathbf{v}^{\dagger} \mathbf{u}=0$ or 1. These will follow as special cases of a more general result (Theorem 5.2). Thus, the inverse is anticausal FIR if and only if $\mathbf{v}^{\dagger} \mathbf{u}=1$ and causal FIR if and only if $\mathbf{v}^{\dagger} \mathbf{u}=0$.

1) Unimodular Systems and Unfactorizability: Consider the $M \times M$ causal FIR system $\mathbf{V}(z)=\mathbf{I}+z^{-1} \mathbf{u} \mathbf{v}^{\dagger}$, where $\mathbf{u}$ and $\mathbf{v}$ are $M \times 1$ vectors. If $\mathbf{v}^{\dagger} \mathbf{u}=0$, it can be verified that the inverse is $\mathbf{I}-z^{-1} \mathbf{u} \mathbf{v}^{\dagger}$; therefore, $\mathbf{V}(z)$ is unimodular. A stronger result is the following.

Lemma 2.1: The system $\mathbf{V}(z)=\mathbf{I}+z^{-1} \mathbf{u v} \mathbf{v}^{\dagger}$ has IIR inverse if $\mathbf{v}^{\dagger} \mathbf{u} \neq 0$ and causal FIR inverse when $\mathbf{v}^{\dagger} \mathbf{u}=0$. Therefore, $\mathbf{V}(z)$ is unimodular if and only if $\mathbf{v}^{\dagger} \mathbf{u}=0$.

Proof: Let $\mathbf{x}_{i}, 1 \leq i \leq M-1$ be a set of independent vectors orthogonal to $\mathbf{v}$. Then, $\mathbf{V}(z) \mathbf{x}_{i}=\mathbf{x}_{i}$. On the other hand, $\mathbf{V}(z) \mathbf{u}=\left(1+z^{-1} \mathbf{v}^{\dagger} \mathbf{u}\right) \mathbf{u}$. Therefore

$$
\mathbf{V}(z)\left[\begin{array}{ll}
\mathbf{u} & \mathbf{P}
\end{array}\right]=\left[\left(1+z^{-1} \mathbf{v}^{\dagger} \mathbf{u}\right) \mathbf{u} \quad \mathbf{P}\right]
$$

where $\mathbf{P}$ is $M \times(M-1)$ with columns equal to $\mathbf{x}_{i}$. If $\mathbf{v}^{\dagger} \mathbf{u} \neq$ 0 , the vector $\mathbf{u}$ is not in the span of $\mathbf{x}_{i}$. Therefore, $\left[\begin{array}{ll}\mathbf{u} & \mathbf{P}\end{array}\right]$ is nonsingular, and we get $[\operatorname{det} \mathbf{V}(z)]=\left(1+z^{-1} \mathbf{v}^{\dagger} \mathbf{u}\right)$. This is not a delay since $\mathbf{v}^{\dagger} \mathbf{u} \neq 0$. Therefore, the inverse of $\mathbf{V}(z)$ is IIR.

More generally, let $\mathbf{G}(z)$ be any degree-one unimodular matrix in $z^{-1}$. Since $\mathbf{G}(\infty)$ is nonsingular, we can always write $\mathbf{G}(z)=\left(\mathbf{I}+z^{-1} \mathbf{u v} \dagger\right) \mathbf{D}$ where $\mathbf{v}^{\dagger} \mathbf{u}=\mathbf{0}$ and $\mathbf{D}=$ $\mathbf{G}(\infty)$.
Example 2.1-A Degree-Two Unfactorizable Unimodular System: We now show that the unimodular system

$$
\mathbf{G}(z)=\left[\begin{array}{cc}
1 & 0 \\
z^{-2} & 1
\end{array}\right]=\left[\begin{array}{ll}
1 & 0 \\
0 & 1
\end{array}\right]+z^{-2}\left[\begin{array}{ll}
0 & 0 \\
1 & 0
\end{array}\right]
$$

cannot be factorized into degree-one causal unimodular systems. Suppose we could then

$$
\mathbf{G}(z)=\left(\mathbf{D}_{0}+z^{-1} \mathbf{u}_{0} \mathbf{v}_{0}^{\dagger}\right)\left(\mathbf{D}_{1}+z^{-1} \mathbf{u}_{1} \mathbf{v}_{1}^{\dagger}\right)
$$

where $\mathbf{D}_{0}$ and $\mathbf{D}_{1}$ are nonsingular and must be such that $\mathbf{D}_{0} \mathbf{D}_{1}=\mathbf{I}$. We can always rearrange this to be of the form $\mathbf{G}(z)=\left(\mathbf{I}+z^{-1} \mathbf{u}_{0} \mathbf{v}_{0}^{\dagger}\right)\left(\mathbf{I}+z^{-1} \mathbf{u}_{1} \mathbf{v}_{1}^{\dagger}\right)$ by redefining the vectors $\mathbf{u}_{i}$ and $\mathbf{v}_{i}$. Comparison of coefficients of $z^{-1}$ in (4) and the product $\left(\mathbf{I}+z^{-1} \mathbf{u}_{0} \mathbf{v}_{0}^{\dagger}\right)\left(\mathbf{I}+z^{-1} \mathbf{u}_{1} \mathbf{v}_{1}^{\dagger}\right)$ shows that we need $\mathbf{u}_{0} \mathbf{v}_{0}^{\dagger}+\mathbf{u}_{1} \mathbf{v}_{1}^{\dagger}=\mathbf{0}$ so that $\mathbf{u}_{1}=c \mathbf{u}_{0}$ for some scalar $c$. This implies $\mathbf{v}_{0}^{\dagger} \mathbf{u}_{1}=0$ since $\mathbf{v}_{0}^{\dagger} \mathbf{u}_{0}=0$ for unimodularity of $\left(\mathbf{I}+z^{-1} \mathbf{u}_{0} \mathbf{v}_{0}^{\dagger}\right)$. Thus, the coefficient of $z^{-2}$ in (5) is $\mathbf{u}_{0}\left(\mathbf{v}_{0}^{\dagger}\right.$ $\left.\mathbf{u}_{1}\right) \mathbf{v}_{1}^{\dagger}=\mathbf{0}$, and the product (5) can never be equal to (4).

\section{B. Degree Reduction Using Degree-one cafacafi Building Blocks}

We are given an $M \times M$ causal FIR matrix $\mathbf{G}_{m}(z)$ with anticausal FIR inverse $\mathbf{H}_{m}(z)$

$$
\mathbf{G}_{m}(z)=\sum_{n=0}^{K} z^{-n} \mathbf{g}_{m}(n), \quad \mathbf{H}_{m}(z)=\sum_{n=0}^{L} z^{n} \mathbf{h}_{m}(n) .
$$

Assume $K, L>0$ and $\mathbf{g}_{m}(K) \neq \mathbf{0}$ and $\mathbf{h}_{m}(L) \neq \mathbf{0}$ to avoid trivialities. Then, $K$ is the order of $\mathbf{G}_{m}(z)$, and $L$ is the order of $\mathbf{H}_{m}(z)$. If the McMillan degree of $\mathbf{G}_{m}(z)$ is $m$, then

$$
\left[\operatorname{det} \mathbf{G}_{m}(z)\right]=c z^{-m}
$$

(see Theorem 5.3 of [1]). Suppose we wish to express it as

$$
\mathbf{G}_{m}(z)=\mathbf{V}_{m}(z) \mathbf{G}_{m-1}(z)
$$

where $\mathbf{V}_{m}(z)$ is a degree-one causal FIR system with anticausal FIR inverse

$$
\mathbf{V}_{m}(z)=\mathbf{I}-\mathbf{u v}^{\dagger}+z^{-1} \mathbf{u} \mathbf{v}^{\dagger}, \quad \mathbf{v}^{\dagger} \mathbf{u}=1 .
$$

See Fig. 3(a). From Theorem 2.1, we have $\left[\operatorname{det} \mathbf{V}_{m}(z)\right]=z^{-1}$ so (7) implies $\left[\operatorname{det} \mathbf{G}_{m-1}(z)\right]=c z^{-(m-1)}$. Therefore, we know that $\mathbf{G}_{m-1}(z)$ has McMillan degree $(m-1)$ as long as it is also causal FIR with anticausal FIR inverse (see Theorem 5.3 of [1]). If we can do this successfully $m$ times, then the final remainder $\mathbf{G}_{0}(z)$ is cafacafi with constant determinant so that it is just a nonsingular constant (see Theorem 5.3 of [1]). This would give the cascaded structure of Fig. 3(b).

The conditions under which we can successfully ensure that $\mathbf{G}_{m-1}(z)$ is cafacafi still need to be explored. Since $\mathbf{V}_{m}^{-1}(z)=\mathbf{V}_{m}\left(z^{-1}\right)$ (Theorem 2.1), we can write

$$
\begin{aligned}
\mathbf{G}_{m-1}(z)= & \left(\mathbf{I}-\mathbf{u} \mathbf{v}^{\dagger}+z \mathbf{u v} \mathbf{v}^{\dagger}\right) \\
& \times\left(\mathbf{g}_{m}(0)+z^{-1} \mathbf{g}_{m}(1)+\cdots+z^{-K} \mathbf{g}_{m}(K)\right) .
\end{aligned}
$$




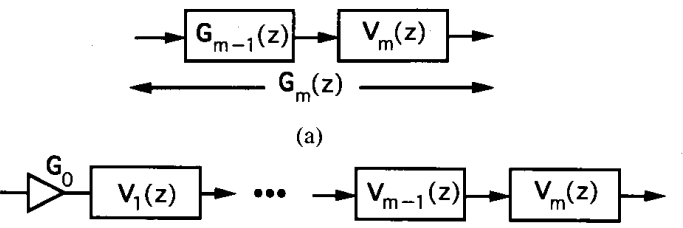

(b)

Fig. 3. (a) Extraction of a cafacafi building block; (b) complete cascaded structure.

Causality of the remainder $\mathbf{G}_{m-1}(z)$ requires $\mathbf{v}^{\dagger} \mathbf{g}_{m}(0)=\mathbf{0}$. Next, consider

$$
\begin{aligned}
\mathbf{G}_{m-1}^{-1}(z)= & \left(\mathbf{h}_{m}(0)+z \mathbf{h}_{m}(1)+\cdots+z^{L} \mathbf{h}_{m}(L)\right) \\
& \times\left(\mathbf{I}-\mathbf{u v} \mathbf{v}^{\dagger}+z^{-1} \mathbf{u v} \mathbf{v}^{\dagger}\right) .
\end{aligned}
$$

Anticausality of this quantity requires $\mathbf{h}_{m}(0) \mathbf{u}=\mathbf{0}$. Summarizing, the degree-reduction procedure will succeed if and only if there exist vectors $\mathbf{u}$ and $\mathbf{v}$ such that

$$
\mathbf{v}^{\dagger} \mathbf{g}_{m}(0)=\mathbf{0}, \quad \mathbf{h}_{m}(0) \mathbf{u}=\mathbf{0}, \quad \mathbf{v}^{\dagger} \mathbf{u}=1 .
$$

We know that $\mathbf{g}_{m}(0)$ and $\mathbf{h}_{m}(0)$ are singular (see Section V$\mathrm{C}$ of [1]), and therefore, there exist nonnull vectors $\mathbf{v}$ and $\mathbf{u}$ satisfying $\mathbf{v}^{\dagger} \mathbf{g}_{m}(0)=\mathbf{0}$ and $\mathbf{h}_{m}(0) \mathbf{u}=\mathbf{0}$. However, there is no guarantee that there will exist $\mathbf{u}$ and $\mathbf{v}$ which are also nonorthogonal (so that they can be scaled to satisfy $\mathbf{v}^{\dagger} \mathbf{u}=1$ ).

In Section VI we will see examples of cafacafi $\mathbf{G}_{m}(z)$ for which (11) cannot be satisfied. In Sections IV-V, we will present some useful subclasses of cafacafi systems for which (11) can be satisfied at every step of the degree reduction process. Towards this goal it proves to be convenient to reformulate the condition (11) in terms of the state space descriptions $(\mathbf{A}, \mathbf{B}, \mathbf{C}, \mathbf{D})$ and $(\hat{\mathbf{A}}, \hat{\mathbf{B}}, \hat{\mathbf{C}}, \hat{\mathbf{D}})$.

\section{State Space Formulation of Factorizability}

In Section III of [1], we described causal systems having anticausal inverses in terms of minimal state space descriptions. Let $(\mathbf{A}, \mathbf{B}, \mathbf{C}, \mathbf{D})$ be a minimal realization of $\mathbf{G}_{m}(z)$. Defining the realization matrix $\mathcal{R}$ and its inverse

$$
\mathcal{R}=\left[\begin{array}{ll}
\mathbf{A} & \mathbf{B} \\
\mathbf{C} & \mathbf{D}
\end{array}\right], \quad\left[\begin{array}{cc}
\hat{\mathbf{A}} & \hat{\mathbf{B}} \\
\hat{\mathbf{C}} & \hat{\mathbf{D}}
\end{array}\right]=\left[\begin{array}{ll}
\mathbf{A} & \mathbf{B} \\
\mathbf{C} & \mathbf{D}
\end{array}\right]^{-1}=\mathcal{R}^{-1}
$$

we obtain the minimal realization $(\hat{\mathbf{A}}, \hat{\mathbf{B}}, \hat{\mathbf{C}}, \hat{\mathbf{D}})$ for the anticausal inverse in the sense defined in Section III-A of [1]. (We have omitted a subscript $m$ on the matrices $(\mathbf{A}, \mathbf{B}, \mathbf{C}, \mathbf{D})$ etc., for simplicity). Note that the inverse of $\mathcal{R}$ exists because of the assumed existence of the anticausal inverse (see Theorem 5.1 of [1]). We can express

$$
\begin{aligned}
& \mathbf{G}_{m}(z)=\mathbf{D}+\sum_{n=1}^{K} z^{-n} \mathbf{C A}^{n-1} \mathbf{B} \\
& \mathbf{H}_{m}(z)=\mathbf{G}_{m}^{-1}(z)=\hat{\mathbf{D}}+\sum_{n=1}^{L} z^{n} \hat{\mathbf{C}} \hat{\mathbf{A}}^{n-1} \hat{\mathbf{B}} .
\end{aligned}
$$

In particular, therefore, $\mathbf{D}=\mathbf{g}_{m}(0)$ and $\hat{\mathbf{D}}=\mathbf{h}_{m}(0)$; therefore, the three conditions in (11) are equivalent to

$$
\mathbf{v}^{\dagger} \mathbf{D}=\mathbf{0}, \quad \hat{\mathbf{D}} \mathbf{u}=\mathbf{0}, \quad \mathbf{v}^{\dagger} \mathbf{u}=1
$$

As stated before, $\mathbf{D}$ and $\mathbf{D}$ are singular, so the only nontrivial issue is to prove the existence of $\mathbf{u}$ and $\mathbf{v}$ such that $v^{\dagger} \mathbf{u}=1$.

In all the results to follow, $(\mathbf{A}, \mathbf{B}, \mathbf{C}, \mathbf{D})$ and $(\hat{\mathbf{A}}, \hat{\mathbf{B}}, \hat{\mathbf{C}}, \hat{\mathbf{D}})$ are minimal realizations of $\mathbf{G}_{m}(z)$ and $\mathbf{G}_{m}^{-1}(z)$, respectively, and are related as in (12). Note that since $\mathbf{G}_{m}(z)$ and $\mathbf{G}_{m}^{-1}(z)$ are FIR, all the eigenvalues of $\mathbf{A}$ and $\hat{\mathbf{A}}$ are equal to zero. By explicitly writing out the four components of the relation $\mathcal{R R}^{-1}=\mathrm{I}$, we obtain the four equations

$$
\begin{array}{ll}
\mathbf{A} \hat{\mathbf{A}}+\mathbf{B} \hat{\mathbf{C}}=\mathbf{I}, & \mathbf{A} \hat{\mathbf{B}}+\mathbf{B} \hat{\mathbf{D}}=\mathbf{0}, \\
\mathbf{C} \hat{\mathbf{A}}+\mathbf{D} \hat{\mathbf{C}}=\mathbf{0}, & \mathbf{C \hat { B }}+\mathbf{D} \hat{\mathbf{D}}=\mathbf{I} .
\end{array}
$$

Similarly, by writing out $\mathcal{R}^{-1} \mathcal{R}=\mathrm{I}$, we get

$$
\begin{array}{ll}
\hat{\mathbf{A}} \mathbf{A}+\hat{\mathbf{B}} \mathbf{C}=\mathbf{I}, & \hat{\mathbf{A}} \mathbf{B}+\hat{\mathbf{B} D}=\mathbf{0}, \\
\hat{\mathbf{C A}}+\hat{\mathbf{D}} \mathbf{C}=\mathbf{0}, & \hat{\mathbf{C B}}+\hat{\mathbf{D} D}=\mathbf{I} .
\end{array}
$$

We will find these equations useful for future reference.

Theorem 3.1: There exist vectors $\mathbf{u}$ and $\mathbf{v}$ satisfying $\mathbf{v}^{\dagger} \mathbf{D}=\mathbf{0}, \hat{\mathbf{D}} \mathbf{u}=\mathbf{0}$, and $\mathbf{v}^{\dagger} \mathbf{u}=1$ if and only if there exist vectors $t$ and $s$ satisfying

$$
\mathbf{A s}=\mathbf{0}, \quad \mathbf{t}^{\dagger} \hat{\mathbf{A}}=\mathbf{0}, \quad \text { and } \quad \mathbf{t}^{\dagger} \mathrm{s}=1 .
$$

Proof: Suppose $\mathbf{v}^{\dagger} \mathbf{D}=\mathbf{0}$ for some v. From (15), we see that this implies $\mathbf{v}^{\dagger} \mathbf{C A}=\mathbf{0}$ and $\mathbf{v}^{\dagger} \mathbf{C B}=\mathbf{v}^{\dagger}$. Defining $\mathbf{t}^{\dagger}=\mathbf{v}^{\dagger} \mathbf{C}$, we see that $\mathbf{t}^{\dagger} \hat{\mathbf{A}}=\mathbf{0}$. Similarly, we can show using (15) that if $\hat{D} \mathbf{u}=\mathbf{0}$ for some $\mathbf{u}$, then $\mathbf{A s}=\mathbf{0}$ where $\mathbf{s}=\hat{\mathbf{B}} \mathbf{u}$. With the quantities $\mathbf{t}$ and $\mathbf{s}$ defined in terms of $\mathbf{v}$ and $\mathbf{u}$ as above, we get

$$
\mathbf{t}^{\dagger} \mathbf{s}=\mathbf{v}^{\dagger} \mathbf{C} \hat{\mathbf{B}} \mathbf{u}=\mathbf{v}^{\dagger} \mathbf{u}
$$

using $\mathbf{C B}=\mathbf{I}-\mathbf{D} \hat{\mathbf{D}}$ (from (15)) and the fact that $\mathbf{v}^{\dagger} \mathbf{D}=\mathbf{0}$. Summarizing, if there exist $\mathbf{u}$ and $\mathbf{v}$ such that $\mathbf{v}^{\dagger} \mathbf{D}=\mathbf{0}$ and $\hat{\mathbf{D}} \mathbf{u}=\mathbf{0}$, then there exist $\mathrm{t}$ and $\mathrm{s}$ such that $\mathrm{t}^{\dagger} \hat{\mathbf{A}}=\mathbf{0}, \mathbf{A s}=\mathbf{0}$, and $\mathbf{t}^{\dagger} \mathbf{s}=\mathbf{v}^{\dagger} \mathbf{u}$.

Second, suppose there exist vectors $\mathbf{s}$ and $\mathbf{t}$ such that $\mathbf{A s}=\mathbf{0}$ and $\mathbf{t}^{\dagger} \hat{\mathbf{A}}=\mathbf{0}$. Defining $\mathbf{u}=\mathbf{C s}$ and $\mathbf{v}^{\dagger}=\mathbf{t}^{\dagger} \hat{\mathbf{B}}$ we can show using (16) that $\hat{D} \mathbf{u}=\mathbf{0}$ and $\mathbf{v}^{\dagger} \mathbf{D}=\mathbf{0}$, and furthermore $\mathbf{v}^{\dagger} \mathbf{u}=\mathbf{t}^{\dagger} \mathbf{s}$. Combining this with the observation in the preceding paragraph, we can say that there exist vectors $\mathbf{u}$ and $\mathbf{v}$ satisfying $\mathbf{v}^{\dagger} \mathbf{D}=\mathbf{0}, \hat{\mathbf{D}} \mathbf{u}=\mathbf{0}$, and $\mathbf{v}^{\dagger} \mathbf{u}=1$ if and only if there exist vectors $\mathbf{t}$ and s satisfying $\mathbf{A s}=\mathbf{0}, \mathbf{t}^{\dagger} \hat{\mathbf{A}}=\mathbf{0}$, and $\mathbf{t}^{\dagger} \mathbf{s}=1$.

In the above theorem we have established a one to one correspondence between the annihilators of the pair $(\mathbf{D}, \hat{\mathbf{D}})$ and the pair $(\mathbf{A}, \hat{\mathbf{A}})$. Therefore, the degree reduction condition for the cafacafi factorization can be reformulated as follows.

Theorem 3.2: The degree reduction step for the causal FIR system $\mathbf{G}_{m}(z)$ with anticausal FIR inverse $\mathbf{G}_{m}^{-1}(z)$ will be successful if and only if there exist vectors $t$ and $s$ satisfying (17) or, equivalently, vectors $\mathbf{u}$ and $\mathbf{v}$ satisfying (14).

1) A Different State-Space Condition: With $\mathbf{G}_{m}(z)$ and $\mathbf{H}_{m}(z)$ expressed as in (6), we know that $\mathbf{h}(0) \mathbf{g}(K)=\mathbf{0}$ and $\mathbf{h}(L) \mathbf{g}(0)=\mathbf{0}$ (subscript $m$ on $\mathbf{g}(n)$ and $\mathbf{h}(n)$ omitted for convenience). This shows that we can satisfy (11) by taking $\mathbf{v}^{\dagger}$ to be any row of $\mathbf{h}(L)$ and $\mathbf{u}$ to be any column of $\mathrm{g}(K)$. There will exist such a choice which further satisfies the condition $\mathbf{v}^{\dagger} \mathbf{u}=1$ as long as $\mathbf{h}(L) \mathbf{g}(K) \neq \mathbf{0}$. In this connection, the following result is helpful. 
Theorem 3.3: Consider the $M \times M$ system $\mathbf{G}_{m}(z)=\sum_{i=0}^{K}$ $z^{-i} \mathbf{g}(i)$ with anticausal FIR inverse $\mathbf{G}_{m}^{-1}(z)=\sum_{i=0}^{L} z^{i} \mathbf{h}(i)$. Let $(\mathbf{A}, \mathbf{B}, \mathbf{C}, \mathbf{D})$ and $(\hat{\mathbf{A}}, \hat{\mathbf{B}}, \hat{\mathbf{C}}, \hat{\mathbf{D}})$ represent their respective minimal realizations related in the usual manner, i.e., as in (12). Then, $\mathbf{h}(L) \mathbf{g}(K)=\mathbf{0}$ if and only if $\hat{\mathbf{A}}^{L-1} \mathbf{A}^{K-1}=\mathbf{0}$.

Proof: We know $\mathbf{h}(L)=\hat{\mathbf{C}} \hat{\mathbf{A}}^{L-1} \hat{\mathbf{B}}$ and $\mathbf{g}(K)=$ $\mathbf{C A}^{K-1} \mathbf{B}$, so that

$$
\begin{aligned}
\mathbf{h}(L) \mathbf{g}(K) & =\hat{\mathbf{C}} \hat{\mathbf{A}}^{L-1} \hat{\mathbf{B}} \mathbf{C} \mathbf{A}^{K-1} \mathbf{B} \\
& =\hat{\mathbf{C}} \hat{\mathbf{A}}^{L-1} \mathbf{A}^{K-1} \mathbf{B}-\hat{\mathbf{C}} \hat{\mathbf{A}}^{L} \mathbf{A}^{K} \mathbf{B} \\
& =\hat{\mathbf{C}} \hat{\mathbf{A}}^{L-1} \mathbf{A}^{K-1} \mathbf{B} .
\end{aligned}
$$

The last equality follows because the FIR property and minimality of the realizations imply $\hat{\mathbf{C}} \hat{\mathbf{A}}^{L}=\mathbf{0}$ and $\mathbf{A}^{K} \mathbf{B}=\mathbf{0}$ (see Lemma 13.9.1 of [4]). Now, consider the product

$$
\underbrace{\left[\begin{array}{c}
\hat{\mathbf{C}} \\
\hat{\mathbf{C}} \hat{\mathbf{A}} \\
\vdots \\
\hat{\mathbf{C}} \hat{\mathbf{A}}^{m-1}
\end{array}\right]}_{\mathbf{P}} \hat{\mathbf{A}}^{L-1} \mathbf{A}^{K-1} \underbrace{\left.\begin{array}{llll}
\mathbf{B} & \mathbf{A B} & \cdots & \mathbf{A}^{m-1} \mathbf{B}
\end{array}\right]}_{\mathbf{Q}}
$$

where $m$ is the McMillan degree of $\mathbf{G}_{m}(z)$. By using $\hat{\mathbf{C}} \hat{\mathbf{A}}^{L}=$ $\mathbf{0}$ and $\mathbf{A}^{K} \mathbf{B}=\mathbf{0}$ it follows that the only nonzero element of this matrix product is the $M \times M$ block matrix $\hat{\mathbf{C}} \hat{\mathbf{A}}^{L-1} \mathbf{A}^{K-1} \mathbf{B}$ (i.e., $\mathbf{h}(L) \mathbf{g}(K)$, by (18)), which will appear on the top left corner. However by minimality we know that $\mathbf{P}$ and $\mathbf{Q}$ have full column-rank and row-rank respectively $(=m)$ so that the above product will be zero if and only if $\hat{\mathbf{A}}^{L-1} \mathbf{A}^{K-1}=\mathbf{0}$. Thus $\mathbf{h}(L) \mathbf{g}(K)=\mathbf{0}$ if and only if $\hat{\mathbf{A}}^{L-1} \mathbf{A}^{K-1}=\mathbf{0}$.

\section{FACTORIZATION OF THE BIORTHOGONAL LAPPED TRANSFORM (BOLT)}

The lapped orthogonal transform (LOT) was introduced in [7] and further studied in [6] and [8]. We will define the LOT to be an $M$ channel maximally decimated analysis bank, in which the polyphase matrix satisfies two properties: first, it is a first-order causal FIR system, that is

$$
\mathbf{G}(z)=\mathbf{g}(0)+z^{-1} \mathbf{g}(1)
$$

(i.e., $\mathbf{E}(z)$ in Fig. 1(b) has the above form). Second, it is paraunitary, that is,

$$
\mathbf{G}^{-1}(z)=\tilde{\mathbf{G}}(z)=\mathbf{g}^{\dagger}(0)+z \mathbf{g}^{\dagger}(1) .
$$

The inverse, therefore, is anticausal FIR. Though $\mathbf{G}(z)$ is a first order system (i.e., the highest power of $z^{-1}$ is $z^{-1}$ ), its degree is equal to the rank of $\mathbf{g}(1)$. Historically, in the original definition of the LOT, the analysis filters were additionally restricted to be symmetric or antisymmetric (i.e., have linear phase) [8]. In our discussion, we shall not make this restriction.

A generalization of the LOT to the biorthogonal case would result if we restrict $\mathbf{G}(z)$ above to be merely FIR with an anticausal FIR inverse, and remove the paraunitary (orthonormal) constraint. The inverse is not necessarily equal to $\tilde{\mathbf{G}}(z)$ anymore. We will call this system the biorthogonal lapped transform (BOLT).

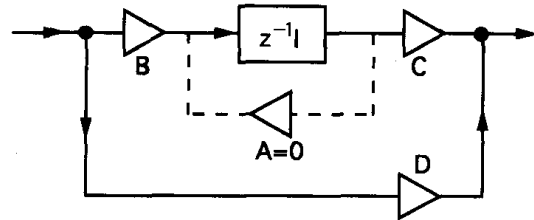

Fig. 4. Implementation of $\mathbf{g}(0)+z^{-1} \mathbf{g}(1)$, where $\mathbf{g}(0)=\mathbf{D}$ and $\mathbf{g}(1)=\mathbf{C B}$.

Definition 4.1-The Biorthogonal Lapped Transform $(B O L T)$ : The BOLT is a maximally decimated analysis bank (Fig. 1), where the polyphase matrix $\mathbf{E}(z)$ is a first-order causal FIR transfer matrix (i.e., as in (20) and has anticausal FIR inverse $\mathbf{G}^{-1}(z)$. We sometimes say that $\mathbf{G}(z)$ is a BOLT matrix. Note that a BOLT matrix is just a first order cafacafi system.

Clearly, the LOT is a special case of the BOLT. Unlike the LOT, the anticausal FIR inverse of the BOLT could have higher order. Here is an example:

$$
\begin{aligned}
\mathbf{G}(z) & =\left[\begin{array}{ccc}
z^{-1} & -1+z^{-1} & 0 \\
0 & 1 & 0 \\
-1+z^{-1} & 0 & z^{-1}
\end{array}\right], \\
\mathbf{G}^{-1}(z) & =\left[\begin{array}{ccc}
z & -1+z & 0 \\
0 & 1 & 0 \\
-z+z^{2} & 1-2 z+z^{2} & z
\end{array}\right],
\end{aligned}
$$

where $\mathbf{G}(z)$ has order $=1$ and the FIR anticausal inverse has order $=2$. However, the degree of $\mathbf{G}^{-1}(z)$ in $z$ is still equal to the degree of $\mathbf{G}(z)$ in $z^{-1}$ (see Observation 5 at the end of Section V-A of [1]).

Let $(\mathbf{A}, \mathbf{B}, \mathbf{C}, \mathbf{D})$ and $(\hat{\mathbf{A}}, \hat{\mathbf{B}}, \hat{\mathbf{C}}, \hat{\mathbf{D}})$ be minimal realizations of $\mathbf{G}(z)$ and its anticausal FIR inverse $\mathbf{G}^{-1}(z)$ related as usual (i.e., (12)). Thus, $\mathbf{g}(0)=\mathbf{D}$, and $\mathbf{g}(1)=\mathbf{C B}$. From the structure shown in Fig. 4, we see that $\mathbf{A}=\mathbf{0}$ for any minimal realization of $\mathbf{G}(z)$. Therefore, any vector $\mathbf{s}$ satisfies $\mathbf{A s}=\mathbf{0}$. Next, all the eigenvalues of $\hat{\mathbf{A}}$ are zero, and there exists $\mathbf{t}^{\dagger} \neq \mathbf{0}$ satisfying $\mathbf{t}^{\dagger} \hat{\mathbf{A}}=\mathbf{0}$. Thus, we can always find vectors $\mathbf{t}$ and s satisfying (17). By using Theorem 3.2, we conclude that the degree reduction step will succeed. ${ }^{2}$ The reduced remainder function will continue to satisfy $\mathbf{A}=\mathbf{0}$ so that we can repeat the degree reduction. We therefore have the following:

Theorem 4.1-BOLT Factorization: Consider an $M$ channel maximally decimated filter bank with analysis bank polyphase matrix $\mathbf{G}(z)=\mathbf{g}(0)+z^{-1} \mathbf{g}(1)$. Suppose this has an FIR anticausal inverse. Then, we can factorize $\mathbf{G}(z)$ as

$$
\mathbf{G}(z)=\mathbf{V}_{\rho}(z) \mathbf{V}_{\rho-1}(z) \cdots \mathbf{V}_{1}(z) \mathbf{G}_{0}
$$

that is, as in Fig. 5, where we have the following:

1) $\rho$ is the McMillan degree of $\mathbf{G}(z)$ (i.e., $\rho=$ the rank of the $M \times M$ matrix $\mathbf{g}(1))$.

2) $\mathbf{V}_{m}(z)=\mathbf{I}-\mathbf{u}_{m} \mathbf{v}_{m}^{\dagger}+z^{-1} \mathbf{u}_{m} \mathbf{v}_{m}^{\dagger}$ with $\mathbf{v}_{m}^{\dagger} \mathbf{u}_{m}=1$.

3) $\mathbf{G}_{0}=\mathbf{G}(1)$ and is nonsingular.

${ }^{2}$ Note that since $\mathbf{A}=\mathbf{0}$, the quantity $\hat{\mathbf{A}}^{L-1} \mathbf{A}^{K-1}=0$ in Theorem 3.3, and yet, the factorization succeeds. This is because $\hat{\mathbf{A}}^{L-1} \mathbf{A}^{K-1} \neq 0$ is only a sufficient but not necessary condition. 


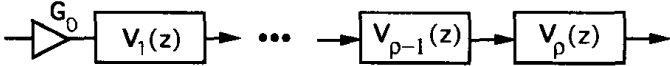

Fig. 5. Factorization of the degree- $\rho$ biorthogonal lapped transform (BOLT) in terms of degree-one cafacafi building blocks.

\section{Comments:}

1) Conversely, a product of the form (22) represents a causal FIR system with anticausal FIR inverse, but it may not be BOLT. This is because in general the product does not have the form (20) but can have higher terms, e.g., $z^{-2} \mathbf{g}(2)$ and so forth. In the next section we will show how to further constrain the parameters of (22) which will ensure that the product is BOLT.

2) If $\mathbf{G}(z)$ has real coefficients it can be verified that the coefficients of $\mathbf{V}_{m}(z)$ are also real.

3) For the special case where the BOLT is a LOT, we have $\mathbf{u}_{m}=\mathbf{v}_{m}$ so that each building block $V_{m}(z)$ is paraunitary.

4) Complexity. Implementation of the building block in Fig. 2 requires $2 M$ multiplications (even if $\mathbf{u}=\mathbf{v}$ as in the LOT case). So the number of multiplications in the structure of Fig. 5 equals $2 M \rho$ plus whatever is required for $\mathbf{G}_{0}$. Since the polyphase matrix works at the decimated rate, this implies we need a total of $2 \rho$ multipliers (plus cost of $\mathbf{G}_{0}$ ) per input sample, to implement the analysis bank of a degree- $\rho$ LOT or BOLT.

5) Smith-McMillan form. With $\rho$ denoting the degree, it can be shown that the Smith-McMillan form of $\mathbf{G}(z)$ is $\boldsymbol{\Lambda}(z)=\left[\begin{array}{cc}z^{-1} \mathbf{I}_{\rho} & \mathbf{0} \\ \mathbf{0} & \mathbf{I}_{M-\rho}\end{array}\right]$. This follows from the fact that the quantities $\ell_{i}$ defined in Theorem 5.2 of [1] satisfy $0 \leq \ell_{i} \leq 1$.

Note that a degree one system also has order $=1$. Therefore, a degree one cafacafi is a BOLT and can be factorized as above, with $\rho=1$. We can express it in the form

$$
\mathbf{G}(z)=\left(\mathbf{I}-\mathbf{u v}^{\dagger}+z^{-1} \mathbf{u} \mathbf{v}^{\dagger}\right) \mathbf{G}_{0}
$$

where $\mathbf{u}^{\dagger} \mathbf{v}=1$ and $\mathbf{G}_{0}$ is nonsingular (in fact $\mathbf{G}_{0}=$ $\mathbf{G}(1)$ ). Therefore, (23) represents the most general degreeone cafacafi. The matrix $\mathbf{G}_{0}$ has $M^{2}$ elements and each of the vectors $\mathbf{u}$ and $\mathbf{v}$ has $M$ elements. Since these elements are constrained by the equationu ${ }^{\dagger} v=1$, the number of degrees of freedom in the degree one cafacafi is equal to $(2 M-1)+M^{2}$. We summarize the preceding result in the following corollary.

Corollary 4.1-The Most General Degree-one cafacafi System: A transfer matrix is cafacafi of degree one if and only if it has the form (23), where $\mathbf{u}^{\dagger} \mathbf{v}=1$, and $\mathbf{G}_{0}$ is nonsingular.

A Second Cascaded Realization: The $M \times M$ building block $\mathbf{V}_{m}(z)$ and the factorization (22) can be rewritten in a form that makes the cafacafi property obvious by inspection. To obtain this, let $\mathbf{u}_{i}, 0 \leq i \leq M-2$ be a set of mutually orthogonal vectors, which in turn are orthogonal to $\mathrm{v}$. We see that $\mathbf{V}(z) \mathbf{u}_{i}=\mathbf{u}_{i}$. (The subscript on $\mathbf{V}(z)$ is dropped for

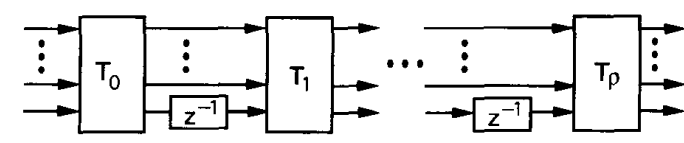

Fig. 6. Second factorization of the BOLT in terms of constant nonsingular matrices.

simplicity.) We also see that $\mathbf{V}(z) \mathbf{u}=z^{-1} \mathbf{u}$. Thus

$$
\begin{aligned}
& \mathbf{V}(z)\left[\begin{array}{lllll}
\mathbf{u}_{0} & \mathbf{u}_{1} & \ldots & \mathbf{u}_{M-2} & \mathbf{u}
\end{array}\right]
\end{aligned}
$$

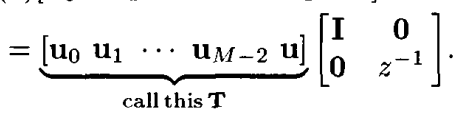

Since $\mathbf{v}^{\dagger} \mathbf{u}=1$, the vector $\mathbf{u}$ is not in the span of the $\mathbf{u}_{i}$ 's. Therefore, the matrix $\mathbf{T}$ is nonsingular and we can rewrite the above as

$$
\mathbf{V}(z)=\mathbf{T}\left[\begin{array}{cc}
\mathbf{I} & \mathbf{0} \\
\mathbf{0} & z^{-1}
\end{array}\right] \mathbf{T}^{-1}
$$

Thus, the general form of the degree-one cafacafi in (23) can be rewritten as

$$
\mathbf{G}(z)=\mathbf{T}\left[\begin{array}{cc}
\mathbf{I} & \mathbf{0} \\
\mathbf{0} & z^{-1}
\end{array}\right] \mathbf{S}
$$

where $\mathbf{T}$ and $\mathbf{S}$ are nonsingular matrices. Therefore, the BOLT factorization (22) can be rewritten as

$$
\mathbf{G}(z)=\mathbf{T}_{\rho}\left[\begin{array}{cc}
\mathbf{I} & \mathbf{0} \\
\mathbf{0} & z^{-1}
\end{array}\right] \mathbf{T}_{\rho-1}\left[\begin{array}{cc}
\mathbf{I} & \mathbf{0} \\
\mathbf{0} & z^{-1}
\end{array}\right] \cdots \mathbf{T}_{1}\left[\begin{array}{cc}
\mathbf{I} & \mathbf{0} \\
\mathbf{0} & z^{-1}
\end{array}\right] \mathbf{T}_{0}
$$

where $\mathbf{T}_{i}$ are nonsingular matrices. The structure is shown in Fig. 6. The previous factorization (22) has only $2 M \rho+M^{2}$ parameters, which is less than the number of matrix elements $M^{2} \rho+M^{2}$ in (27). Therefore, there is some redundancy in the representation (27), but its advantage is that it is explicitly clear that the inverse is anticausal FIR.

\section{MORE Results on FIRST-ORdER SySTEMS AND BIORTHOGONAL LAPPED TRANSFORMS}

Consider a first order $M \times M$ transfer matrix of the form

$$
\mathbf{G}(z)=\mathbf{g}(0)+z^{-1} \mathbf{g}(1) .
$$

Let the McMillan degree be $\rho$ (i.e., the rank of $\mathbf{g}(1)$ is $\rho$ ). Suppose $\mathbf{G}(1)$ is nonsingular (as is the case when there exists an FIR inverse, since the determinant would then be a delay). We can then rewrite $\mathbf{G}(z)=\mathbf{G}(1) \mathbf{F}(z)$ where $\mathbf{F}(1)=\mathbf{I}$. Therefore, we can write

$$
\mathbf{F}(z)=\mathbf{I}-\mathcal{U} \mathcal{V}^{\dagger}+z^{-1} \underbrace{\mathcal{U}}_{M \times \rho \rho \times M} \underbrace{\mathcal{V}^{\dagger}}
$$

with the constant matrices $\mathcal{U}$ and $\mathcal{V}^{\dagger}$ having rank $\rho$. We will now relate the properties of the inverse $\mathbf{F}^{-1}(z)$ to the properties of the matrices $\mathcal{U} \mathcal{V}^{\dagger}$ and $\mathcal{V}^{\dagger} \mathcal{U}$. Such a study adds significantly to the understanding of the biorthogonal lapped transform. 


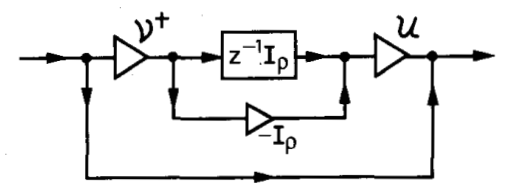

Fig. 7. Implementation of the system $\mathbf{F}(z)$.

\section{A. Inverse of the First-Order System $\left(\mathbf{I}-\mathcal{U} \mathcal{V}^{\dagger}+z^{-1} \mathcal{U} \mathcal{V}^{\dagger}\right)$}

The nature of the inverse of (29) depends largely on the properties of the $\rho \times \rho$ matrix $\mathcal{V}^{\dagger} \mathcal{U}$ as shown by the results to be developed below.

Lemma 5.1: Consider the system $\mathbf{F}(z)=\mathbf{I}-\mathcal{U V}^{\dagger}+$ $z^{-1} \mathcal{U} \mathcal{V}^{\dagger}$, where $\mathcal{U}$ and $\mathcal{V}$ are $M \times \rho$ with rank $\rho$ (so that $\mathbf{F}(z)$ has degree $\rho$ ). There exists an anticausal inverse for this system if and only if $\mathcal{V}^{\dagger} \mathcal{U}$ (which is $\rho \times \rho$ ) is nonsingular.

Proof: Fig. 7 shows an implementation of $\mathbf{F}(z)$ with $\rho$ delays, i.e., a minimal implementation. The state space description $(\mathbf{A}, \mathbf{B}, \mathbf{C}, \mathbf{D})$ for this is

$$
\mathbf{A}=\mathbf{0}, \mathbf{B}=\mathcal{V}^{\dagger}, \mathbf{C}=\mathcal{U}, \mathbf{D}=\mathbf{I}-\mathcal{U V}^{\dagger} .
$$

The realization matrix $\mathcal{R}$ is then

$$
\mathcal{R}=\left[\begin{array}{ll}
\mathbf{A} & \mathbf{B} \\
\mathbf{C} & \mathbf{D}
\end{array}\right]={ }_{M}^{\rho}\left(\begin{array}{cc}
\rho & M \\
\mathbf{0} & \mathcal{V}^{\dagger} \\
\mathcal{U} & \mathbf{I}-\mathcal{U} \mathcal{V}^{\dagger}
\end{array}\right) .
$$

Recall from Theorem 5.1 of [1] that there exists an anticausal inverse if and only if the above matrix is nonsingular. We will show that this matrix is nonsingular if and only if $\mathcal{V}^{\dagger} \mathcal{U}$ is nonsingular. Suppose $\mathcal{R} \mathbf{x}=\mathbf{0}$ for some vector $\mathbf{x}=\left[\begin{array}{l}\mathbf{x}_{1} \\ \mathbf{x}_{2}\end{array}\right]$. Then

$$
\mathcal{V}^{\dagger} \mathbf{x}_{2}=\mathbf{0}, \quad \text { and } \quad \mathcal{U} \mathbf{x}_{1}+\mathbf{x}_{2}=\mathbf{0}
$$

Combining these two equations, we get $\mathcal{V}^{\dagger} \mathcal{U} \mathbf{x}_{1}=0$. If $\mathcal{V}^{\dagger} \mathcal{U}$ is nonsingular, then $x_{1}=0$, and therefore, $x_{2}=-\mathcal{U} x_{1}=0$ from (32). This implies that if $\mathcal{R} \mathbf{x}=0$, then $\mathbf{x}$ is necessarily 0 . Therefore, $\mathcal{R}$ is nonsingular.

On the other hand, if $\mathcal{V}^{\dagger} \mathcal{U}$ is singular, there exists $\mathbf{y} \neq \mathbf{0}$ such that $\mathcal{V}^{\dagger} \mathcal{U}_{\mathbf{y}}=\mathbf{0}$. If we now choose $\mathbf{x}_{1}=-\mathbf{y}$ and $\mathbf{x}_{2}=\mathcal{U} \mathbf{y}$, then $\mathcal{R}$ is annihilated by $\mathbf{x}$ proving that it is singular. Therefore, $\mathcal{R}$ is nonsingular if and only if $\mathcal{V}^{\dagger} \mathcal{U}$ is nonsingular. This completes the proof.

As an example suppose

$$
\mathcal{U V}^{\dagger}=\left[\begin{array}{l}
1 \\
1
\end{array}\right]\left[\begin{array}{ll}
1 & -1
\end{array}\right]
$$

Then, $\rho=1$, but $\mathcal{V}^{\dagger} \mathcal{U}=0$, therefore, there does not exist an anticausal inverse. As another example suppose $\mathcal{U V}^{\dagger}$ itself is nonsingular (i.e., $\rho=M$ ); then $\mathcal{V}^{\dagger} \mathcal{U}$ is nonsingular and there exists an anticausal inverse, possibly IIR. The next theorem makes precise the conditions under which the inverses are FIR.
Theorem 5.1: Consider the first-order system $\mathbf{F}(z)=\mathbf{I}-$ $\mathcal{U} \mathcal{V}^{\dagger}+z^{-1} \mathcal{U} \mathcal{V}^{\dagger}$, where $\mathcal{U}$ and $\mathcal{V}$ are $M \times \rho$ with rank $\rho$ (so $\mathcal{U} \mathcal{V}^{\dagger}$ has rank $\rho$ and $\mathbf{F}(z)$ has degree $\rho$ ). Then, the inverse of $\mathbf{F}(z)$ is

1) FIR if and only if all eigenvalues of $\mathcal{U} \mathcal{V}^{\dagger}$ are restricted to be 0 's and 1 's.

2) FIR and anticausal (i.e., $\mathbf{F}(z)$ is cafacafi) if and only if $\mathcal{U N}^{\dagger}$ has $\rho$ of its eigenvalues equal to unity and the remaining $M-\rho$ eigenvalues equal to zero.

3) FIR and causal (i.e., $\mathbf{F}(z)$ is unimodular in $z^{-1}$ ) if and only if $\mathcal{U V}^{\dagger}$ has all eigenvalues equal to zero.

Comments:

1) Restricting the eigenvalues of a matrix $\mathbf{P}$ to be zeros and ones does not imply that $\mathbf{P}^{2}=\mathbf{P}$ or that it is a projection matrix. ${ }^{3}$ For example, the matrix $\mathcal{U V}^{\dagger}$ in (33) has all eigenvalues $=0$, but $\mathbf{P}^{2}=\mathbf{0} \neq \mathbf{P}$.

2) Since $\mathcal{U} \mathcal{V}^{\dagger}$ has rank $\rho$, it can have at most $\rho$ nonzero eigenvalues. However, it could be fewer, as in the extreme example of a triangular matrix with all diagonal elements equal to zero. Another example is (33), which has rank $=1$, but all the eigenvalues are equal to zero.

Proof of Theorem 5.1: From the unitary triangularization theorem [11], we can write $\mathcal{U} \mathcal{V}^{\dagger}=\mathbf{T} \Delta \mathbf{T}^{\dagger}$, where $\mathbf{T} \mathbf{T}^{\dagger}=\mathbf{I}$, and $\boldsymbol{\Delta}$ is upper triangular with the eigenvalues $\left\{\lambda_{0}, \lambda_{1} \cdots \lambda_{\rho-1}, 0, \ldots 0\right\}$ on the diagonals. (Since the rank is $\rho$ there could be at most $\rho$ nonzero eigenvalues). We can then express

$$
\mathbf{F}(z)=\mathbf{T}\left(\mathbf{I}-\boldsymbol{\Delta}+z^{-1} \boldsymbol{\Delta}\right) \mathbf{T}^{\dagger}
$$

so that

$$
\operatorname{det} \mathbf{F}(z)=\prod_{i=0}^{\rho-1}\left(1-\lambda_{i}+z^{-1} \lambda_{i}\right) .
$$

This is of the form $c z^{-K}$ (which is necessary and sufficient for the existence of an FIR inverse) if and only if $\lambda_{i}=$ 0 or 1 for each $i$. Since the degree of $\mathbf{F}(z)$ is $\rho$, the FIR inverse is anticausal if and only if the determinant is $c z^{-\rho}[1$, theorem 5.3]. This will be the case if and only if $\mathcal{U} \mathcal{V}^{\dagger}$ has $\rho$ eigenvalues equal to unity (and, of course, the remaining $M-\rho$ eigenvalues $=0$ ). Finally the FIR inverse is causal (i.e., $\mathbf{F}(z)$ is unimodular) if and only if the determinant is a constant, that is $\lambda_{i}=0$ for all $i$.

We can combine Lemma 5.1 and Theorem 5.1 and restate everything in terms of $\mathcal{V}^{\dagger} \mathcal{U}$ rather than $\mathcal{U} \mathcal{V}^{\dagger}$ as follows.

Theorem 5.2: Consider the system $\mathbf{F}(z)=\mathbf{I}-\mathcal{U V}^{\dagger}+$ $z^{-1} \mathcal{U} \mathcal{V}^{\dagger}$, where $\mathcal{U}$ and $\mathcal{V}$ are $M \times \rho$ with rank $\rho$ (so $\mathcal{U} \mathcal{V}^{\dagger}$ has rank $\rho$ and $\mathbf{F}(z)$ has degree $\rho$ ). Then

1) $\mathbf{F}(z)$ has an anticausal inverse if and only if $\mathcal{V}^{\dagger} \mathcal{U}$ is nonsingular.

2) The inverse of $\mathbf{F}(z)$ is FIR if and only if all eigenvalues of $\mathcal{V}^{\dagger} \mathcal{U}$ are restricted to be 0 's and 1 's.

${ }^{3}$ A matrix $\mathbf{P}$ is said to be a projection if it is Hermitian and $\mathbf{P}^{2}=\mathbf{P}$ (see p. 75 of $[10])$. 
3) The inverse of $\mathbf{F}(z)$ is FIR and anticausal (i.e., $\mathbf{F}(z)$ is cafacafi) if and only if $\mathcal{V}^{\dagger} \mathcal{U}$ has all eigenvalues equal to unity.

4) The inverse of $\mathbf{F}(z)$ is FIR and causal (i.e., $\mathbf{F}(z)$ is unimodular) if and only if $\mathcal{V}^{\dagger} \mathcal{U}$ has all eigenvalues equal to zero.

Proof: Part 1 is a repetition of Lemma 5.1. Parts 2 and 4 follow from Theorem 5.1 by using the fact that every nonzero eigenvalue of the matrix $\mathbf{P Q}$ is an eigenvalue of $\mathbf{Q P}$ (for any two matrices $\mathbf{P}$ and $\mathbf{Q}$ for which $\mathbf{P Q}$ and $\mathbf{Q P}$ are defined). Part 3 follows by combining parts 1 and 2 ; indeed, the nonsingularity of $\mathcal{V}^{\dagger} \mathcal{U}$ and the condition that the eigenvalues be restricted to be ones and zeros is equivalent to the statement that all the eigenvalues of $\mathcal{V}^{\dagger} \mathcal{U}$ are equal to unity.

Example 5.1: The cases where $\mathcal{V}^{\dagger} \mathcal{U}=\mathbf{I}_{\rho}$ and $\mathcal{V}^{\dagger} \mathcal{U}=\mathbf{0}$ give examples of FIR systems with anticausal and causal FIR inverses, respectively. We have

$$
\begin{aligned}
(\mathbf{I} & \left.-\mathcal{U} \mathcal{V}^{\dagger}+z^{-1} \mathcal{U} \mathcal{V}^{\dagger}\right)^{-1} \\
& = \begin{cases}\mathbf{I}-\mathcal{U} \mathcal{V}^{\dagger}+z \mathcal{U} \mathcal{V}^{\dagger} & \text { for } \mathcal{V}^{\dagger} \mathcal{U}=\mathbf{I}_{\rho} \\
\mathbf{I}+\mathcal{U} \mathcal{V}^{\dagger}-z^{-1} \mathcal{U} \mathcal{V}^{\dagger} & \text { for } \mathcal{V}^{\dagger} \mathcal{U}=0\end{cases}
\end{aligned}
$$

as one can verify by direct multiplication. Notice that if $\mathcal{V}^{\dagger} \mathcal{U}=\mathbf{I}_{\rho}$ then the inverse is also of first order. Therefore, first order cafacafi systems with higher order inverses (as in (21)) are not covered by the system with $\mathcal{V}^{\dagger} \mathcal{U}=\mathbf{I}_{\rho}$. In Section II-A, we saw the special case where $\rho=1$ (i.e., $\mathcal{U}$ and $\mathcal{V}$ were vectors with $\mathcal{V}^{\dagger} \mathcal{U}=1$ and 0 , respectively).

Example 5.2: The following example

$$
\mathcal{U V}^{\dagger}=\left[\begin{array}{ll}
1 & 0 \\
0 & 0 \\
0 & 1
\end{array}\right]\left[\begin{array}{lll}
0 & 1 & 0 \\
1 & 0 & 0
\end{array}\right], \quad \mathcal{V}^{\dagger} \mathcal{U}=\left[\begin{array}{ll}
0 & 0 \\
1 & 0
\end{array}\right]
$$

satisfies part 4 of Theorem 5.2 so that $\mathrm{F}(z)$ is unimodular, even though $\mathcal{V}^{\dagger} \mathcal{U} \neq 0$.

Example 5.3: As a special case, consider $\mathbf{I}-\mathbf{P}+z^{-1} \mathbf{P}$ where $\mathbf{P}^{2}=\mathbf{P}$. With $\rho$ denoting the rank of $\mathbf{P}$, we can write $\mathbf{P}=\mathcal{U} \mathcal{V}^{\dagger}$. Now $\mathbf{P}^{2}=\mathbf{P}$ implies $\mathcal{U V}^{\dagger} \mathcal{U} \mathcal{V}^{\dagger}=\mathcal{U} \mathcal{V}^{\dagger}$. Premultiplying by $\mathcal{U}^{\dagger}$ and postmultiplying with $\mathcal{V}$ and using the facts that $\mathcal{U}^{\dagger} \mathcal{U}$ and $\mathcal{V}^{\dagger} \mathcal{V}$ are nonsingular we obtain $\mathcal{V}^{\dagger} \mathcal{U}=$ I. From part 3 of Theorem 5.2 we therefore conclude that there exists an anticausal FIR inverse for $\mathbf{I}-\mathbf{P}+z^{-1} \mathbf{P}$, when $\mathbf{P}^{2}=\mathbf{P}$. In fact the inverse is $\mathbf{I}-\mathbf{P}+z \mathbf{P}$, as can be verified by direct substitution.

Example 5.4-Unimodular System: By a slight modification of the above theorem we can show that $\mathbf{I}+z^{-1} \mathcal{U} \mathcal{V}^{\dagger}$ is unimodular if and only if $\mathcal{V}^{\dagger} \mathcal{U}$ has all eigenvalues equal to zero.

\section{B. General Expression and Complete Parameterization of the Biorthogonal Lapped Transforms (BOLT)}

In Section IV, we considered the biorthogonal lapped transforms or BOLT systems. These are first-order cafacafi systems, that is, systems of the form $\mathbf{G}(z)=\mathbf{g}(0)+z^{-1} \mathbf{g}(1)$ with anticausal FIR inverses. Since this implies $\mathbf{G}(1)$ is nonsingular, we can write $\mathbf{G}(z)=\mathbf{G}(1) \mathbf{F}(z)$ where $\mathbf{F}(z)$ is as in (29). Using
Theorem 5.2 (part 3) we can say that a system $\mathrm{G}(z)$ is BOLT if and only if it has the form $\mathbf{G}(z)=\mathbf{G}(1)\left(\mathbf{I}-\mathcal{U} \mathcal{V}^{\dagger}+z^{-1} \mathcal{U} \mathcal{V}^{\dagger}\right)$, where $\mathcal{V}^{\dagger} \mathcal{U}$ has all eigenvalues equal to unity.

In Section IV, we showed that the BOLT can be factorized as in (22), where

$$
\mathbf{V}_{m}(z)=\mathbf{I}-\mathbf{u}_{m} \mathbf{v}_{m}^{\dagger}+z^{-1} \mathbf{u}_{m} \mathbf{v}_{m}^{\dagger}, \quad \mathbf{v}_{m}^{\dagger} \mathbf{u}_{m}=1
$$

and $\rho$ is the degree of $\mathbf{G}(z)$ (i.e., $\rho=$ rank of $\mathbf{g}(1)$ ). Conversely, if we have a product of the form (22) with $\mathbf{V}_{m}(z)$ as above, it still represents a system with anticausal FIR inverse, but may have order $>1$ (i.e., there could be terms $\mathbf{g}(n) z^{-n}, n>1$ in $\left.\mathbf{G}(z)\right)$. To ensure that the product has order $=1$ (i.e., that it represents a BOLT), we need to impose further restrictions on $\mathbf{u}_{k}$ and $\mathbf{v}_{k}$. Suppose we restrict these vectors to be such that

$$
\mathbf{v}_{k}^{\dagger} \mathbf{u}_{i}=\left\{\begin{array}{ll}
0, & 1 \leq i \leq k-1 \\
1, & i=k
\end{array} .\right.
$$

Then, it is easily verified by induction that the product

$$
\mathbf{G}(z)=\mathbf{G}(1) \mathbf{V}_{\rho}(z) \mathbf{V}_{\rho-1}(z) \cdots \mathbf{V}_{1}(z)
$$

with $\mathbf{V}_{m}(z)$ defined as above does reduce to the form

$$
\mathbf{G}(z)=\mathbf{G}(1)\left(\mathbf{I}-\mathcal{U V}^{\dagger}+z^{-1} \mathcal{U} \mathcal{V}^{\dagger}\right)
$$

with the constant matrices $\mathcal{V}$ and $\mathcal{U}$ given by

$$
\mathcal{V}=\left[\begin{array}{llll}
\mathbf{v}_{1} & \mathbf{v}_{2} & \cdots & \mathbf{v}_{\rho}
\end{array}\right], \quad \mathcal{U}=\left[\begin{array}{llll}
\mathbf{u}_{1} & \mathbf{u}_{2} & \cdots & \mathbf{u}_{\rho}
\end{array}\right]
$$

Notice that the constant matrix G(1) occurs as the left-most factor unlike in (22). This difference is immaterial; a slight variation of the steps would lead to the form (22). Except for this difference, the structure for (39) is as in Fig. 5.

Conversely, can we represent any BOLT system as in (39) with the restriction (38)? The answer is in the affirmative: If $\mathbf{G}(z)$ is BOLT, this means in particular that it has an FIR inverse, and therefore, $\mathbf{G ( 1 )}$ is nonsingular. Therefore, we can always write a degree $\rho$ BOLT as in (40), where $\mathcal{U}$ and $\mathcal{V}$ are $M \times \rho$ matrices with rank $\rho$. Now, $\mathcal{U} \mathcal{V}^{\dagger}=\mathcal{U} \mathbf{T} \mathbf{T}^{\dagger} \mathcal{V}^{\dagger}$ for any unitary $T$, and we can rewrite $\mathcal{U} \mathcal{V}^{\dagger}=\mathcal{U}_{1} \mathcal{V}_{1}^{\dagger}$ by defining $\mathcal{U}_{1}=$ $\mathcal{U} \mathbf{T}$ and $\mathcal{V}_{1}^{\dagger}=\mathbf{T}^{\dagger} \mathcal{V}^{\dagger}$. Note that $\mathcal{V}_{1}^{\dagger} \mathcal{U}_{1}=\mathbf{T}^{\dagger} \mathcal{V} \mathcal{V}^{\dagger} \mathcal{U} \mathbf{T}$. By proper choice of $\mathbf{T}$, we can ensure that $\mathcal{V}_{1}^{\dagger} \mathcal{U}_{1}$ is a triangular matrix. In other words, we can assume without loss of generality that $\mathcal{V}^{\dagger} \mathcal{U}$ is triangular. Since $\mathbf{G}(z)$ is cafacafi, we see that this matrix has all diagonal elements equal to unity (use part 3 of Theorem 5.2), that is

$$
\mathcal{V}^{\dagger} \mathcal{U}=\left[\begin{array}{ccccc}
1 & \times & \times & \ldots & \times \\
0 & 1 & \times & \ldots & \times \\
0 & 0 & 1 & \ldots & \times \\
\vdots & \vdots & \vdots & \ddots & \vdots \\
0 & 0 & 0 & \ldots & 1
\end{array}\right]
$$

where $x$ stands for possibly nonzero elements. Now, denote the columns of $\mathcal{V}$ and $\mathcal{U}$ as in (41). Then the property (42) means that $(38)$ is satisfied. 
Thus, we have defined a set of vectors $\mathbf{u}_{m}$ and $\mathbf{v}_{m}, 1 \leq$ $m \leq \rho$ such that they satisfy (38). We already mentioned that if such $\mathbf{u}_{m}$ and $\mathbf{v}_{m}$ are used in the product (39), the result has the form (40) with $\mathcal{U}$ and $\mathcal{V}$ given by (41). In other words, the given BOLT matrix (40) can indeed be represented as in (39), with the vectors satisfying (38).

We can summarize all of the above results as follows.

Theorem 5.3-BOLT Characterization: Consider an $M \times$ $M$ transfer matrix $\mathbf{G}(z)$. We say that this is a BOLT if $\mathbf{G}(z)=\mathbf{g}(0)+z^{-1} \mathbf{g}(1)$, and it has an anticausal FIR inverse. The following statements are equivalent:

1) $\mathbf{G}(z)$ is a BOLT.

2) $\mathbf{G}(z)$ can be factorized as $\mathbf{G}(z)=\mathbf{G}(1) \mathbf{V}_{\rho}(z) \mathbf{V}_{\rho-1}(z)$ $\ldots \mathbf{V}_{1}(z)$, where $\mathbf{G}(1)$ is nonsingular and $\mathbf{V}_{m}(z)$ are as in (37), with the vectors $\mathbf{v}_{k}$ and $\mathbf{u}_{i}$ satisfying (38).

3) $\mathbf{G}(z)$ can be written in the form $\mathbf{G}(z)=\mathbf{G}(1)(\mathbf{I}-$ $\left.\mathcal{U} \mathcal{V}^{\dagger}+z^{-1} \mathcal{U} \mathcal{V}^{\dagger}\right)$, where $\mathbf{G}(1)$ is nonsingular and $\mathcal{V}^{\dagger} \mathcal{U}$ has all eigenvalues equal to unity.

4) $\mathbf{G}(z)$ can be written in the form $\mathbf{G}(z)=\mathbf{G}(1)(\mathbf{I}-$ $\left.\mathcal{U V}^{\dagger}+z^{-1} \mathcal{U V}^{\dagger}\right)$, where $\mathbf{G}(1)$ is nonsingular and $\mathcal{V}^{\dagger} \mathcal{U}$ has the triangular form (42).

Thus, if $\mathrm{G}(z)$ is BOLT with $\mathcal{V}^{\dagger} \mathcal{U}$ written in the form (42), the columns of $\mathcal{V}$ and $\mathcal{U}$ (see (41)) satisfy (38) and can be taken to be the vectors $\mathbf{v}_{m}$ and $\mathbf{u}_{m}$ in the factorization (39). Therefore, the factorization is determined simply by identifying the columns of $\mathcal{V}$ and $\mathcal{U}$.

Degrees of Freedom: Thus, the BOLT is characterized by (39) which has a nonsingular matrix $\mathbf{G}(1)$ with $M^{2}$ elements, and $2 \rho$ vectors $\mathbf{u}_{k}, \mathbf{v}_{k}$, with $M$ elements each, that is, there are $M^{2}+2 \rho M$ scalar elements associated with the expression (39). However, the number of freedoms is less than this, in view of the constraints (38). In the real coefficient case, it can be verified that the $M \times M$ degree- $\rho$ BOLT has $M^{2}+2 \rho M-$ $0.5 \rho(\rho+1)$ degrees of freedom. In the special case of the LOT (i.e., the paraunitary case), we have $\mathbf{u}_{k}=\mathbf{v}_{k}$ and $\mathbf{G}(1)$ is unitary so there are only $0.5 M(M-1)+\rho M-0.5 \rho(\rho+1)$ degrees of freedom. Traditional transform coding (which is a special case where $\mathcal{U} \mathcal{V}^{\dagger}=\mathbf{0}$ and $\mathbf{G}(1)$ is unitary) has only $0.5 M(M-1)$ freedoms. The extra freedom offered by the BOLT can perhaps be exploited to obtain better attenuation for the analysis filters (see example below), or to impose other constraints such as linear phase, regularity (for wavelet synthesis [12]) and so forth. This topic requires detailed investigation, and is beyond the scope of this paper.

Design Example 5.1-The BOLT Filter Bank: We now present a design example for the BOLT filter bank. ${ }^{4}$ Let $M=8$ (i.e., an eight channel filter bank; see Fig. 1(a)). Let the polyphase matrix $\mathbf{E}(z)=\mathbf{g}(0)+z^{-1} \mathbf{g}(1)$ with rank of $\mathrm{g}(1)$ equal to three (i.e., degree of $\mathbf{E}(z)$ is three). This is constrained to be a BOLT by expressing it in the factored form (39) and constraining the vectors to satisfy (38). Under these constraints, the magnitude responses $\left|H_{k}\left(e^{j \omega}\right)\right|$ of the analysis filters are optimized. The result is shown in Fig. 8(a).

For comparison, Fig. 8(b) shows the responses of the corresponding LOT filter bank (i.e., with the vectors further

\footnotetext{
${ }^{4}$ We would like to thank Y.-P. Lin, graduate student, Caitech, for generating this example.
}

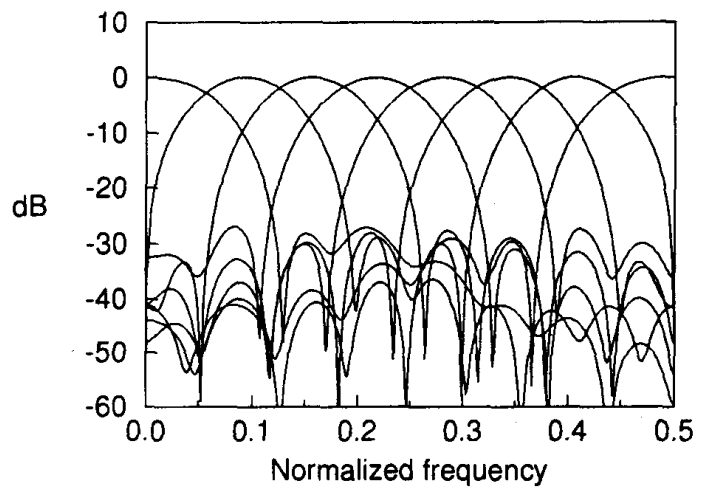

(a)

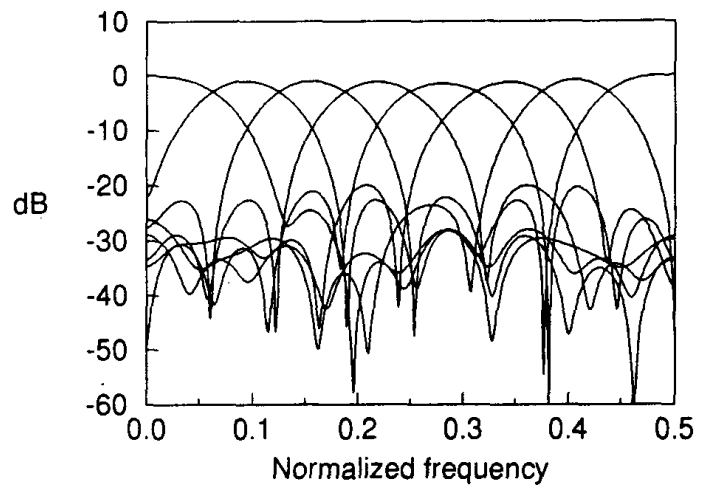

(b)

Fig. 8. Design Example 5.1. Magnitude responses of the analysis filters for an eight-channel filter bank: (a) BOLT filter bank; (b) LOT filter bank. The degree of the polyphase matrix is $\rho=3$.

constrained such that $\mathbf{v}_{i}=\mathbf{u}_{i}$ for each $i$, and $G(1)$ constrained to be unitary). The improved filtering characteristics of the BOLT over the LOT is clear from the plots. The BOLT provides a minimum stopband attenuation of $27 \mathrm{~dB}$, whereas it is about $20 \mathrm{~dB}$ for the LOT. Notice that the analysis filters in the examples do not necessarily have linear phase; our definitions of the LOT and the BOLT do not impose this restriction.

\section{DEGREE-Two DyAdIC BuILDING Blocks}

If the degree-one reduction scheme of Section II has to work, there should exist vectors $u$ and $v$ such that (11) holds. If this is not the case, one might consider extracting the building block from the right rather than left, i.e., one might try the decomposition $\mathbf{G}_{m}(z)=\mathbf{G}_{m-1}(z) \mathbf{V}_{m}(z)$ instead of (7). In this case, the degree reduction equations remain the same except that $\mathbf{g}_{m}(0)$ and $\mathbf{h}_{m}(0)$ are interchanged. Thus, degree-one reduction will fail when neither of the following two conditions:

Condition 1: $\quad \mathbf{v}^{\dagger} \mathbf{g}_{m}(0)=\mathbf{0}, \quad \mathbf{h}_{m}(0) \mathbf{u}=\mathbf{0}, \quad \mathbf{u}^{\dagger} \mathbf{v}=1$,

Condition 2: $\quad \mathbf{v}^{\dagger} \mathbf{h}_{m}(0)=\mathbf{0}, \quad \mathbf{g}_{m}(0) \mathbf{u}=\mathbf{0}, \quad \mathbf{u}^{\dagger} \mathbf{v}=1$

can be satisfied for any choice of $u$ and $v$. 


\section{A. Cases Where Degree-One Reduction Fails}

We can create examples of cafacafi systems for which degree-one reduction will fail. For example, let us consider the $2 \times 2$ case $(M=2)$. In this case, we can exactly specify the conditions when the degree reduction will fail.

Lemma 6.1: Let $\mathbf{G}_{m}(z)$ be $2 \times 2$ cafacafi with inverse $\mathbf{H}_{m}(z)$ (both as in (6)). Assume $\mathbf{g}_{m}(0) \neq 0$ and $\mathbf{h}_{m}(0) \neq 0$ and $K, L>0$ in (6). Then, the degree reduction by one (using the building block (2) will fail if and only if $g_{m}(0) \mathbf{h}_{m}(0)$ $=\mathbf{h}_{m}(0) \mathbf{g}_{m}(0)=\mathbf{0}$, that is, if and only if $\mathbf{D D}=\hat{\mathbf{D D}}=\mathbf{0}$ in terms of state space notations (Section III).

Proof: Since the $2 \times 2$ matrices $g_{m}(0)$ and $h_{m}(0)$ are singular (see Section V-C of [1]) and nonzero, the vectors $\mathbf{v}$ and $\mathbf{u}$ satisfying $\mathbf{v}^{\dagger} \mathbf{h}_{m}(0)=\mathbf{0}$ and $\mathbf{g}_{m}(0) \mathbf{u}=\mathbf{0}$ are unique up to scale. Clearly, $\mathbf{g}_{m}(0)$ and $h_{m}(0)$ have rank one, and we can write

$$
\mathbf{g}_{m}(0)=\mathbf{a b} \mathbf{b}^{\dagger} \text { and } \mathbf{h}_{m}(0)=\mathbf{c d}^{\dagger}
$$

for some $2 \times 1$ nonnull vectors $\mathbf{a}, \mathbf{b}, \mathbf{c}$ and $\mathbf{d}$. Thus, $\mathbf{v}^{\dagger} \mathbf{h}_{m}(0)=\mathbf{0}$ and $\mathbf{g}_{m}(0) \mathbf{u}=\mathbf{0}$ imply, respectively

$$
\mathbf{v}^{\dagger} \mathbf{c}=\mathbf{0} \text { and } \mathbf{b}^{\dagger} \mathbf{u}=\mathbf{0} \text {. }
$$

From this, we see that if $\mathbf{v}^{\dagger} \mathbf{u}=0$, then $\mathbf{u}=\mathbf{c}$ and $\mathbf{v}=\mathbf{b}$ (up to scale), and this implies $\mathbf{b}^{\dagger} \mathbf{c}=\mathbf{v}^{\dagger} \mathbf{u}=0$, that is, $\mathbf{g}_{m}(0)$ $\mathbf{h}_{m}(0)=\mathbf{0}$. Therefore, if (44) cannot be satisfied then $\mathbf{g}_{m}(0)$ $\mathbf{h}_{m}(0)=\mathbf{0}$. Conversely, let $\mathbf{g}_{m}(0) \mathbf{h}_{m}(0)=\mathbf{0}$. Then, $\mathbf{b}^{\dagger} \mathbf{c}=$ 0 . Therefore, the conditions $\mathbf{v}^{\dagger} \mathbf{h}_{m}(0)=\mathbf{0}$ and $\mathbf{g}_{m}(0) \mathbf{u}=\mathbf{0}$ imply, respectively, $\mathbf{v}=\mathbf{b}$ and $\mathbf{u}=\mathbf{c}$ (up to scale) so that $\mathbf{v}^{\dagger} \mathbf{u}=\mathbf{b}^{\dagger} \mathbf{c}=0$, and (44) cannot be satisfied. Thus, (44) cannot be satisfied if and only if $\mathbf{g}_{m}(0) \mathbf{h}_{m}(0)=0$. Similarly, (43) cannot be satisfied if and only if $\mathbf{h}_{m}(0) \mathbf{g}_{m}(0)=0$.

Since the cafacafi system and its inverse satisfy the state space relations (15) and (16), we can restate the above result in terms of state space parameters (Section III) like this: the degree-one reduction step will fail in the $2 \times 2$ case if and only if

$$
\hat{\mathbf{C B}}=\mathbf{C} \hat{\mathbf{B}}=\mathbf{I} \text {. }
$$

This follows by setting $\hat{\mathbf{D} D}=\mathbf{0}$ in the last equation of (16) and $\mathbf{D D}=\mathbf{0}$ in the last equation of (15).

1) $2 \times 2$ Example Where Degree-One Reduction Fails: Now, consider the $M \times M$ system

$$
\mathbf{G}_{m}(z)=\mathbf{a} \mathbf{b}^{\dagger}+z^{-1} \mathbf{I}+z^{-2} \mathbf{a} \mathbf{b}^{\dagger}, \quad \mathbf{a}^{\dagger} \mathbf{b}=0 .
$$

where $\mathrm{a}$ and $\mathrm{b}$ are nonzero $M \times 1$ vectors. We can verify that the inverse is $\mathbf{G}_{m}^{-1}(z)=-\mathbf{a b} \mathbf{b}^{\dagger}+z \mathbf{I}-\mathbf{a b}^{\dagger} z^{2}$ by multiplying the two expressions. This system is therefore cafacafi. Since $\mathbf{a}^{\dagger} \mathbf{b}=0$, we have $\mathbf{g}_{m}(0) \mathbf{h}_{m}(0)=\mathbf{h}_{m}(0) \mathbf{g}_{m}(0)=\mathbf{0}$. Therefore, by Lemma 6.1 , the degree reduction step will fail in the $M=2$ case. For $M=2$, the system (45) therefore serves as a cafacafi example where the degree-one reduction fails, that is, neither (43) nor (44) be satisfied for any pair of vectors $\mathbf{u}$ and $\mathbf{v}$.

In Appendix A, we show that for arbitrary $M$ the degree of (45) is equal to $M$, and present a minimal implementation (Fig. 10).

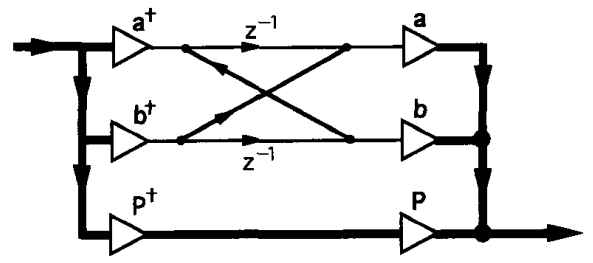

Fig. 9. Minimal structure for the $M \times M$ degree-2 cafacafi system (46)

2) $M \times M$ Example Where Degree-One Reduction Fails: If $M>2$, the system in (45) is still cafacafi, but its degree can be reduced successfully by one, using the building block (2). To see this, note that in this case there exists a vector $\mathbf{w}$ orthogonal to both $\mathbf{a}$ and $\mathbf{b}$ so that we can set $\mathbf{u}=\mathbf{v}=\mathbf{w}$ and satisfy (43). To create an $M \times M$ example that cannot be factorized into degree-one building blocks, consider

$$
\mathbf{G}(z)=\mathbf{P} \mathbf{P}^{\dagger}+\mathbf{a} \mathbf{b}^{\dagger}+z^{-1}\left(\mathbf{I}-\mathbf{P P}^{\dagger}\right)+z^{-2} \mathbf{a} \mathbf{b}^{\dagger}
$$

where $\mathbf{P}$ is $M \times(M-2)$, and $\mathbf{a}$ and $\mathbf{b}$ are column vectors such that $[\mathbf{P} \mathbf{a} \mathbf{b}]$ is unitary. It can then be verified that its inverse is

$$
\mathbf{G}^{-1}(z)=\mathbf{P} \mathbf{P}^{\dagger}-\mathbf{a b}^{\dagger}+z\left(\mathbf{I}-\mathbf{P P}^{\dagger}\right)-z^{2} \mathbf{a} \mathbf{b}^{\dagger}
$$

which is anticausal FIR. In the notation of (6), we have $\mathbf{g}_{m}(0)=\mathbf{P P}^{\dagger}+\mathbf{a b} \mathbf{b}^{\dagger}$ and $\mathbf{h}_{m}(0)=\mathbf{P P}^{\dagger}-\mathbf{a} \mathbf{b}^{\dagger}$. Both of these matrices have rank $M-1$ (e.g., note that $\mathbf{g}_{m}(0)[\mathbf{P} \mathbf{a} \mathbf{b}]=$ $\left[\begin{array}{lll}\mathbf{P} & \mathbf{0} & \mathbf{a}\end{array}\right]$; therefore, $\mathbf{g}_{m}(0)$ has rank $\left.M-1\right)$ so that the annihilating vectors $\mathbf{u}$ and $\mathbf{v}$ in (43) are unique. In fact the annihilating vectors in (43) are $\mathbf{u}=\mathbf{a}$ and $\mathbf{v}=\mathbf{b}$ so that $\mathbf{u}^{\dagger} \mathbf{v}=0$. Thus, the condition $\mathbf{u}^{\dagger} \mathbf{v}=1$ in (43) cannot be satisfied. Similarly (44) cannot be satisfied. Therefore, the degree of (46) cannot be reduced by extracting a degree-one cafacafi building block.

The degree of $\mathbf{G}(z)$ is clearly $\geq 2$ since the order is seen to be two from (46). We will show that the degree is exactly two by displaying an implementation with two delays. Since $[\mathbf{P} \quad \mathbf{a} \quad \mathbf{b}]$ is unitary, we have $\mathbf{I}_{M}=\mathbf{a a}^{\dagger}+\mathbf{b b}^{\dagger}+\mathbf{P P}^{\dagger}$. Using this, we can see that the system $G(z)$ in (46) can be implemented as in Fig. 9. Therefore, the degree of $\mathbf{G}(z)$ is two indeed.

\section{B. Degree Reduction Equations with Degree-Two Building Blocks}

The fact that we cannot factorize the $2 \times 2$ system (45) into degree-one blocks leads us to ask if we can factorize a general $2 \times 2$ cafacafi system using a combination of degreeone and degree-two building blocks. With some algebra it can be shown (Appendix B) that the most general $2 \times 2$ cafacaf system with degree equal to two, which cannot be factorized into degree one cafacafi systems has the form

$$
\mathbf{v}_{2}(z)=\mathbf{u} \mathbf{v}^{\dagger}+z^{-1} \mathbf{I}+s z^{-2} \mathbf{u} \mathbf{v}^{\dagger}, \quad \mathbf{u}^{\dagger} \mathbf{v}=0
$$

where $s$ is a nonzero scalar. ${ }^{5}$ By explicit multiplication we can verify that the inverse is $\mathbf{V}_{2}^{-1}(z)=-s \mathbf{u v} \mathbf{v}^{\dagger}+z \mathbf{I}-z^{2} \mathbf{u v} \mathbf{v}^{\dagger}$.

${ }^{5}$ We can of course multiply this with a nonsingular constant matrix, but it can be absorbed in $\mathbf{G}_{m-2}(z)$ in (49) and is of no interest. 
Since $\mathrm{V}_{2}(z)$ is degree-two cafacafi, we have $\left[\operatorname{det} \mathrm{V}_{2}(z)\right]=$ $c z^{-2}, c \neq 0$

Now, let $\mathbf{G}_{m}(z)$ be degree-m cafacafi with $\left[\operatorname{detG}_{m}(z)\right]=$ $c_{m} z^{-m}$. Suppose degree-one reduction fails (i.e., we cannot find $\mathbf{u}$ and $\mathbf{v}$ satisfying either (43) or (44)). Suppose we wish to use (48) to obtain a degree reduction by two, i.e., we wish to find a degree- $(m-2)$ cafacafi system $\mathbf{G}_{m-2}(z)$ such that

$$
\mathbf{G}_{m}(z)=\mathbf{V}_{2}(z) \mathbf{G}_{m-2}(z) .
$$

Since $\left[\operatorname{det} \mathbf{V}_{2}(z)\right]=c z^{-2}$, we have $\left[\operatorname{det} \mathbf{G}_{m-2}(z)\right]=$ $c_{m-2} z^{-(m-2)}$. It can be shown (Appendix C) that $\mathbf{G}_{m-2}(z)$ will be cafacafi if and only if $\mathbf{u}$ and $\mathbf{v}$ are such that

$$
\mathbf{g}_{m}(0)=\mathbf{u} \mathbf{v}^{\dagger} \mathbf{g}_{m}(1), \quad \mathbf{h}_{m}(0)=-s \mathbf{h}_{m}(1) \mathbf{u v} \mathbf{v}^{\dagger}
$$

where $\mathbf{g}_{m}(n)$ and $\mathbf{h}_{m}(n)$ are the impulse response coefficients of $\mathbf{G}_{m}(z)$ and its inverse respectively (see (6)). If the above can be satisfied by choice of $\mathbf{u}$ and $\mathbf{v}$ then $\mathbf{G}_{m-2}(z)$ is cafacafi with degree $m-2$ because its determinant is $c_{m-2} z^{-(m-2)}$ (see Theorem 5.3 of [1]).

1) Another Example of an Irreducible cafacafi System: Consider the $2 \times 2$ system $\mathbf{G}_{4}(z)=\mathbf{a} \mathbf{b}^{\dagger}+z^{-2} \mathbf{I}+z^{-4} \mathbf{a b}{ }^{\dagger}$ with $\mathbf{a}^{\dagger} \mathbf{b}=0$, which is the same as (45) with $z$ replaced by $z^{2}$. Therefore, it is cafacafi. We still have $\mathrm{g}_{m}(0)=\mathbf{a b} \mathbf{b}^{\dagger}$ and $\mathbf{h}_{m}(0)=-\mathbf{a b} \mathbf{b}^{\dagger}$ so that degree-one reduction is not possible (as seen in Section VI-A). Since $\mathrm{g}_{m}(1)=\mathbf{0},(50)$ cannot be satisfied. Thus we cannot do degree reduction by two, if we use the building block (48). Since the degree one building block and the degree two building block used above are the most general cafacafi building blocks, the system $\mathbf{G}_{4}(z)$ cannot be factorized into lower degree cafacafi blocks at all.

\section{FACTORIZATION OF CAUSAL} FIR SYSTEMS HAVING FIR INVERSE

Let $\mathbf{G}_{m}(z)$ be a causal FIR system, with an FIR inverse (not necessarily anticausal). Then its determinant has the form $c z^{-m}$, though $m$ does not represent the McMillan degree unless the inverse is anticausal. Unless $\mathbf{G}_{m}(z)$ is unimodular in $z^{-1}$, we have $m>0$, and the determinant is zero for $z=\infty$. In other words, the constant coefficient $\mathbf{g}_{m}(0)=\mathbf{G}_{m}(\infty)$ is singular.

Suppose we wish to express $\mathbf{G}_{m}(z)$ in the form $\mathbf{G}_{m}(z)=$ $\mathbf{V}_{m}(z) \mathbf{G}_{m-1}(z)$, where $\mathbf{V}_{m}(z)$ is the familiar cafacafi building block (8). Since $\mathbf{G}_{m-1}(z)=\mathbf{V}_{m}\left(z^{-1}\right) \mathbf{G}_{m}(z)$, it is still FIR. From (9), we see that we can force it to be causal by choosing $\mathbf{v}$ such that $\mathbf{v}^{\dagger} \mathbf{g}_{m}(0)=0$. The singularity of $\mathbf{g}_{m}$ (0) ensures the existence of such nonnull $\mathbf{v}$. The choice of $\mathbf{u}$ is arbitrary except for the requirement $\mathbf{u}^{\dagger} \mathbf{v}=1$ in (8). For example, we can make $\mathbf{u}=\mathbf{v}$ with unit norm in which case $\mathbf{V}_{m}(z)$ becomes paraunitary.

Since $\left[\operatorname{det} \mathbf{V}_{m}(z)\right]=z^{-1}$, we have $\left[\operatorname{det} G_{m-1}(z)\right]=$ $c z^{-(m-1)}$. Thus $\mathbf{G}_{m-1}(z)$ is causal and FIR with the degree of determinant reduced by one. We can repeat this process until we obtain

$$
\mathbf{G}_{m}(z)=\mathbf{V}_{m}(z) \mathbf{V}_{m-1}(z) \cdots \mathbf{V}_{1}(z) \mathbf{G}_{0}(z)
$$

where $\mathbf{G}_{0}(z)$ is unimodular (causal and FIR with determinant $c \neq 0$ ). Therefore, we have proved the following.
Theorem 7.1: Let $\mathbf{G}_{m}(z)$ be $M \times M$ causal FIR with FIR inverse so that $\left[\operatorname{det}_{m}(z)\right]=c z^{-m}, c \neq 0$. Then, we can factorize it as in (51), where $\mathbf{V}_{m}(z)=\left(\mathbf{I}-\mathbf{u}_{m} \mathbf{v}_{m}^{\dagger}+z^{-1}\right.$ $\left.\mathbf{u}_{m} \mathbf{v}_{m}^{\dagger}\right), \mathbf{v}_{m}^{\dagger} \mathbf{u}_{m}=1$, and $\mathbf{G}_{0}(z)$ is unimodular in $z^{-1}$. The matrices $\mathbf{V}_{m}(z)$ can be chosen to be paraunitary if desired (by taking $\mathbf{u}_{m}=\mathbf{v}_{m}$ ) in which case the product of quantities on the right hand side preceding $\mathbf{G}_{0}(z)$ is paraunitary.

This result has some resemblance to the so called inner-outer factorization used in system theory [1, ref. [35]]. However, the preceding statement is for FIR matrices with FIR inverses and provides detailed structural form for the factorization. As in Section IV, we can replace the building blocks $\mathbf{V}_{i}(z)$ as in (25) to obtain a factorization of the form (27), where $\mathbf{T}_{0}$ is replaced with a unimodular remainder $\mathbf{G}_{0}(z)$. For the special case where $\mathbf{G}_{m}(z)$ is paraunitary with degree $m$, the building blocks $\mathbf{V}_{i}(z)$ are paraunitary, and the terminator $\mathbf{G}_{0}(z)$ becomes a unitary constant. In this case all $\mathbf{T}_{i}$ in (27) will be unitary.

We therefore see that any casual FIR system $\mathbf{G}_{N}(z)$ with an FIR inverse can be written as $\mathbf{G}_{N}(z)=\mathbf{G}_{c, a}(z) \mathbf{G}_{c, c}(z)$, where $\mathbf{G}_{c, a}(z)$ is causal FIR with anticausal FIR inverse, and $\mathbf{G}_{c, c}(z)$ is causal FIR with causal FIR inverse. This follows by letting $\mathbf{G}_{0}(z)=\mathbf{G}_{c, c}(z)$ and lumping the remaining factors on the right side of (51) into $\mathbf{G}_{c, a}(z)$. In particular we can let $\mathbf{G}_{c, a}(z)$ be paraunitary without loss of generality. Notice, however, that the degree of $\mathbf{G}_{m}(z)$ is not, in general, the sum of the degrees of $\mathbf{G}_{c, a}(z)$ and $\mathbf{G}_{c, c}(z)$; therefore, this is not a minimal decomposition.

\section{Concluding Remarks and Open Problems}

Many of the previously reported designs for perfect reconstruction filter banks were orthonormal (i.e., the polyphase matrix $\mathrm{E}(z)$ was paraunitary). In the IIR case this meant that if the analysis filters are causal and stable (poles inside the unit circle) then the synthesis filters would be anticausal and stable (poles outside the unit circle). In [1], we argued that for the FIR case, the more general class of biorthogonal systems can be characterized if we can characterize all causal FIR polyphase matrices with anticausal FIR inverse (i.e., all cafacafi matrices). More generally, the relevance of systems with anticausal inverses was elaborated in Section I-A of [1].

The basic similarity between causal systems with anticausal inverses and causal paraunitary systems is fascinating. First, the latter is a special case of the former. Second, the former is characterized by nonsingular realization matrices (for minimal realizations) whereas the latter is characterized by unitary realization matrices (up to similarity). Finally, in the FIR case, both of these classes have determinant equal to $c z^{-N}$, where $N$ is the McMillan degree. (That is, both of them achieve the maximum value that the degree of a determinant can ever achieve, viz., the McMillan degree.) In both cases, the most general degree-one FIR building block has the form $\left(\mathbf{I}-\mathbf{u} \mathbf{v}^{\dagger}+z^{-1} \mathbf{u v} \mathbf{v}^{\dagger}\right) \mathbf{G}_{0}$, where $\mathbf{u}^{\dagger} \mathbf{v}=1$, and $\mathbf{G}_{0}$ is nonsingular. In the paraunitary case, we further have $u=v$, and $\mathbf{G}_{0}$ is unitary. 


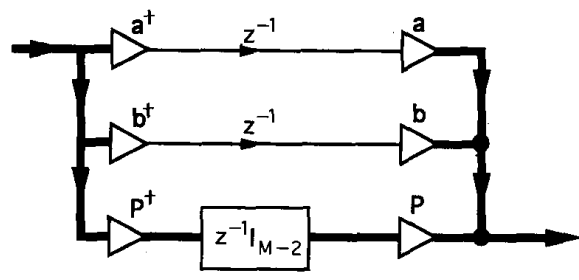

(a)

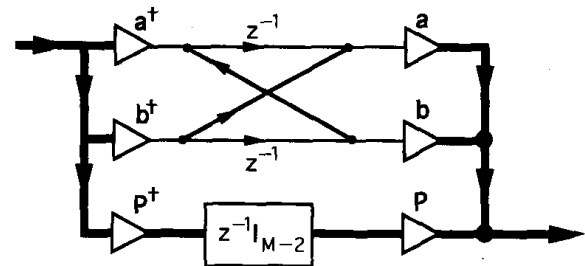

(b)

Fig. 10. (a) Conceptual implementation of $z^{-1} \mathbf{I}_{M}$; (b) implementation of the degree- $M$ system given by $\mathbf{a b}^{\dagger}+z^{-1} \mathbf{I}_{M}+z^{-2} \mathbf{a b}^{\dagger}$.

The most significant difference between causal systems with anticausal inverses and causal paraunitary systems is that the former cannot in general be factorized into degree one building blocks whereas the latter can be so factorized. This factorization was used in the past (see references in ch. 6 of [4]) for the design and implementation of orthonormal perfect reconstruction filter banks. We saw in Section IV that a special case of cafacafi systems can indeed be factorized into degreeone cafacafi building blocks. These are cafacafi systems of order one. This factorization gives rise to the biorthogonal lapped transform (BOLT) which is a generalization of the lapped orthogonal transform LOT.

The BOLT is a maximally decimated analysis bank where the polyphase matrix is a first order causal FIR system with anticausal inverse. Since it is a generalization of the lapped orthogonal transform, it provides additional degrees of freedom in the design. It remains to see how to exploit this freedom while designing filter banks for data compression, or for generation of biorthogonal wavelets and so forth. These require further investigation. There are other problems requiring further investigation. In this paper we introduced two cafacafi building blocks, namely the degree one building block (8) and the degree two building block (48). (These are the most general building blocks we need to consider). We showed that a subclass of cafacafi systems, namely the BOLT system can be factorized using degree-one building blocks. On the other hand the degree two building block (48) cannot be expressed as a product of the degree-one building blocks. Furthermore there exist examples of cafacafi systems whose degree cannot be reduced using either of these two building blocks (see the end of Section VI-B), that is, they cannot be expressed as a product of any combination of the two building blocks.

However, what does that mean? Perhaps there is a broader class of building blocks which will suffice for factorization; perhaps the number of required building blocks somehow depends on the order and the size $(M \times M)$ of the cafacafi matrices. This seems to be an open issue requiring deeper investigation.

In principle the set of all cafacafi matrices can be characterized in terms of the realization matrix $\mathcal{R}$ (see (12)). For cafacafi systems, the matrix $\mathcal{R}$ is invertible, $\mathbf{A}$ has all eigenvalues equal to zero (equivalently $\mathbf{A}^{N}=0$ where $\mathbf{A}$ is $N \times N$ ), and furthermore the matrix $\hat{\mathbf{A}}$ in the inverse (12) has all eigenvalues equal to zero. Therefore, $M \times M$ cafacafi matrices with degree $N$ are completely characterized by the set of all $(N+M) \times(N+M)$ matrices $\mathcal{R}$ having the following properties:

i) They are nonsingular.

ii) The top-left $N \times N$ submatrix $\mathbf{A}$ has all eigenvalues equal to zero.

iii) The top-left $N \times N$ submatrix $\hat{\mathbf{A}}$ of $\mathcal{R}^{-1}$ has all eigenvalues equal to zero.

Finding a simple analytic way to impose these three restrictions on a constant $(N+M) \times(N+M)$ matrix $\mathcal{R}$ is still an open problem.

\section{APPENDIX A}

DEGREE OF THE SECOND-ORDER SYSTEM (45)

For arbitrary $M$, the system (45) has degree $M$. To see this, first consider $\mathbf{U}(z) \triangleq \mathbf{I}+z^{-1} \mathbf{a} \mathbf{b}^{\dagger}$, with $\mathbf{a}^{\dagger} \mathbf{b}=0$. This is unimodular (i.e., $[\operatorname{det} \mathbf{U}(z)]=c \neq 0$ ) with $\mathbf{U}^{-1}(z)=$ $\mathbf{I}-\mathbf{a} \mathbf{b}^{\dagger} z^{-1}$. Clearly, the causal FIR system $z^{-1} \mathbf{U}(z)$ has the anticausal FIR inverse $z \mathbf{I}-\mathbf{a b ^ { \dagger }}$, and by construction, its determinant is $c z^{-M}$. In other words, $z^{-1} \mathrm{U}(z)$ is cafacafi and its degree is $M$ (see Theorem 5.3 of [1]). The system (45), which is $\mathbf{a} \mathbf{b}^{\dagger}+z^{-1} \mathbf{U}(z)$ therefore has degree $M$.

How do we find a structure for $\mathbf{G}(z)$ with only $M$ delays? Since $\mathbf{a}$ and $\mathbf{b}$ are mutually orthogonal vectors, we can define a $M \times M$ unitary matrix of the form $[\mathbf{P} \mathbf{a} \mathbf{b}]$ where $\mathbf{P}$ is $M \times(M-2)$. (For this purpose we assume that $\mathbf{a}$ and $\mathbf{b}$ have unit norm for simplicity.) We then have $\mathbf{I}_{M}=\mathbf{a a}^{\dagger}+\mathbf{b b}^{\dagger}+$ $\mathbf{P} \mathbf{P}^{\dagger}$ so that we can implement $z^{-1} \mathbf{I}_{M}$ as shown in Fig. 10(a). If we insert two new branches as shown in Fig. 10(b), we obtain a realization of (45) with $M$ delays. This, therefore, is a minimal realization.

\section{APPENDIX B}

Most General Degree-Two BuILding Block for $M=2$

We now find the most general $2 \times 2$ degree-two cafacafi system $\mathbf{G}(z)$ that cannot be factorized into degree-one building blocks. Since $\mathbf{G}(z)$ has degree $=2$, it has the form

$$
\mathbf{G}(z)=\mathbf{g}(0)+z^{-1} \mathbf{g}(1)+z^{-2} \mathbf{g}(2) \text {. }
$$

If $\mathbf{g}(2)=\mathbf{0}$, this becomes a BOLT and can be factorized (Section IV); therefore, we must have $\mathbf{g}(2) \neq \mathbf{0}$. If $\mathbf{g}(0)=\mathbf{0}$, then the degree-reduction condition (11) is trivially satisfied because we can first choose $\mathbf{u}$, and then, let $\mathbf{v}=\mathbf{u}$. Summarizing, we have $\mathbf{g}(0) \neq \mathbf{0}$ and $\mathbf{g}(2) \neq \mathbf{0}$.

We know that $\mathbf{G}^{-1}(z)$ has degree two in $z$ (see Section V-A of [1]). Therefore, it has the form $\mathbf{H}(z)=\mathbf{h}(0)+$ $z \mathbf{h}(1)+z^{2} \mathbf{h}(2)$. Since $\mathbf{G}(z)$ cannot be factorized into degree one cafacafi systems, we cannot factorize $\mathbf{H}\left(z^{-1}\right)$ into degree 
one cafacafi systems. Therefore, we can modify the argument in the preceding paragraph and obtain $\mathbf{h}(0) \neq \mathbf{0}$ and $\mathbf{h}(2) \neq \mathbf{0}$. If we now equate the like powers of $z$ in $\mathbf{G}(z) \mathbf{H}(z)=\mathbf{I}$, we obtain, among other things, $\mathbf{g}(0) \mathbf{h}(2)=\mathbf{0}$ and $\mathbf{g}(2) \mathbf{h}(0)=\mathbf{0}$. Since none of the matrices is null and all of them are $2 \times$ 2 , this implies that they all have rank one. Therefore, we can write

$$
\begin{aligned}
\mathbf{G}(z) & =\mathbf{u} \mathbf{v}^{\dagger}+z^{-1} \mathbf{g}(1)+z^{-2} \mathbf{x} \mathbf{y}^{\dagger} \\
\mathbf{G}^{-1}(z) & =\mathbf{H}(z)=\mathbf{v}_{\perp} \mathbf{u}_{\perp}^{\dagger}+z \mathbf{h}(1)+z^{2} \mathbf{y}_{\perp} \mathbf{x}_{\perp}^{\dagger}
\end{aligned}
$$

for some nonzero vectors $\mathbf{u}, \mathbf{v}, \mathbf{x}, \mathbf{y}, \mathbf{u}_{\perp}, \mathbf{v}_{\perp}, \mathbf{x}_{\perp}$ and $\mathbf{y}_{\perp}$.

From Lemma 6.1, we know that in the $2 \times 2$ case, failure of degree-one reduction implies $\mathbf{h}(0) \mathbf{g}(0)=\mathbf{g}(0) \mathbf{h}(0)=\mathbf{0}$. Therefore, we conclude that $\mathbf{v}^{\dagger} \mathbf{v}_{\perp}=0$ and $\mathbf{u}_{\perp}^{\dagger} \mathbf{u}=0$ (hence, the notation with subscript $\perp$ ). Now, the condition $\mathbf{G}(z) \mathbf{H}(z)=\mathbf{I}$ implies, in particular, that $\mathbf{v}^{\dagger} \mathbf{y}_{\perp}=0$ and $\mathbf{y}^{\dagger} \mathbf{v}_{\perp}=0$ (since $z^{2}$ and $z^{-2}$ terms in the product are zero). Since all vectors are $2 \times 1$ and nonnull, we conclude $y_{\perp}=v_{\perp}$ and $\mathbf{y}=\mathbf{v}$ up to scale. Similarly from $\mathbf{H}(z) \mathbf{G}(z)=\mathbf{I}$ we conclude that $\mathbf{x}=\mathbf{u}$ and $\mathbf{x}_{\perp}=\mathbf{u}_{\perp}$ up to scale. Summarizing, the two matrices must have the form

$$
\begin{aligned}
& \mathbf{G}(z)=\mathbf{u} \mathbf{v}^{\dagger}+z^{-1} \mathbf{g}(1)+z^{-2} s_{1} \mathbf{u} \mathbf{v}^{\dagger} \\
& \mathbf{H}(z)=\mathbf{v}_{\perp} \mathbf{u}_{\perp}^{\dagger}+z \mathbf{h}(1)+z^{2} s_{2} \mathbf{v}_{\perp} \mathbf{u}_{\perp}^{\dagger}
\end{aligned}
$$

for nonzero scalars $s_{1}, s_{2}$. Since $\mathbf{v}^{\dagger} \mathbf{v}_{\perp}=\mathbf{u}^{\dagger} \mathbf{u}_{\perp}=0$, we see that the condition $\mathbf{G}(z) \mathbf{H}(z)=\mathbf{I}$ implies $\mathbf{g}(1) \mathbf{h}(1)=\mathbf{I}$. Therefore, $\mathbf{g}(1)$ and $\mathbf{h}(1)$ are nonsingular. We can always factor them out, so let us assume $\mathbf{g}(1)=\mathbf{h}(1)=\mathbf{I}$, that is, except for a constant nonsingular factor, we have the form

$$
\begin{aligned}
& \mathbf{G}(z)=\mathbf{u v}^{\dagger}+z^{-1} \mathbf{I}+z^{-2} s_{1} \mathbf{u v}^{\dagger} \\
& \mathbf{H}(z)=\mathbf{v}_{\perp} \mathbf{u}_{\perp}^{\dagger}+z \mathbf{I}+z^{2} s_{2} \mathbf{v}_{\perp} \mathbf{u}_{\perp}^{\dagger}
\end{aligned}
$$

for nonzero scalars $s_{1}, s_{2}$. Now, by equating the coefficients of $z$ in $\mathbf{G}(z) \mathbf{H}(z)=\mathbf{I}$ we get $\mathbf{u} \mathbf{v}^{\dagger}=-s_{2} \mathbf{v}_{\perp} \mathbf{u}_{\perp}^{\dagger}$. Similarly $\mathbf{v}_{\perp} \mathbf{u}_{\perp}^{\dagger}=-s_{\perp} \mathbf{u} \mathbf{v}^{\dagger}$. This means, in particular, $\mathbf{u}=\mathbf{v}_{\perp}$ up to scale, and therefore $\mathbf{u}^{\dagger} \mathbf{v}=0$. Summarizing, the most general $2 \times 2$ degree-two cafacafi system $\mathbf{G}(z)$, which cannot be factorized into degree-one building blocks has the form

$$
\mathbf{G}(z)=\left(\mathbf{u} \mathbf{v}^{\dagger}+z^{-1} \mathbf{I}+z^{-2} s_{1} \mathbf{u} \mathbf{v}^{\dagger}\right) \mathbf{g}(1)
$$

where $\mathbf{u}^{\dagger} \mathbf{v}=0, \mathbf{g}(1)$ is arbitrary nonsingular, and $s_{1}$ is arbitrary but nonzero. Its anticausal FIR inverse is

$$
\mathbf{H}(z)=[\mathbf{g}(1)]^{-1}\left(-s_{1} \mathbf{u} \mathbf{v}^{\dagger}+z \mathbf{I}-z^{2} \mathbf{u} \mathbf{v}^{\dagger}\right)
$$

as we can double check by multiplying $\mathbf{G}(z)$ and $\mathbf{H}(z)$.

\section{APPENDIX $\mathrm{C}$ \\ DEgREE Two REDUCTION}

Given the cafacafi system $\mathbf{G}_{m}(z)$ with inverse $\mathbf{H}_{m}(z)$ as in (6), suppose we wish to perform a degree reduction by two, using the cafacafi building block $V_{2}(z)$ in (48). This means that we wish to find the vectors $\mathbf{u}$ and $\mathbf{v}$ such that $\mathbf{G}_{m}(z)$ can be expressed as in (49) where $\mathbf{G}_{m-2}(z)$ is cafacafi. Since $\mathbf{G}_{m-2}(z)=\mathbf{V}_{2}^{-1}(z) \mathbf{G}_{m}(z)$ it is clear that it is already FIR, and so is $\mathbf{G}_{m-2}^{-1}(z)=\mathbf{G}_{m}^{-1}(z) \mathbf{V}_{2}(z)$. Only causality of $\mathbf{G}_{m-2}(z)$ and anticausality of $\mathbf{G}_{m-2}^{-1}(z)$ need to be enforced by choice of $u$ and $v$. We have

$$
\begin{aligned}
\mathbf{G}_{m-2}(z)= & \mathbf{V}_{2}^{-1}(z) \mathbf{G}_{m}(z) \\
= & \left(-s \mathbf{u} \mathbf{v}^{\dagger}+z \mathbf{I}-z^{2} \mathbf{u} \mathbf{v}^{\dagger}\right) \\
& \times\left(\mathbf{g}_{m}(0)+z^{-1} \mathbf{g}_{m}(1)+\ldots\right) .
\end{aligned}
$$

Causality of this requires that the coefficients of $z$ and $z^{2}$ be zero, that is, $\mathbf{g}_{m}(0)-\mathbf{u v}^{\dagger} \mathbf{g}_{m}(1)=\mathbf{0}$, and $\mathbf{u v} \mathbf{v}^{\dagger} \mathbf{g}_{m}(0)=\mathbf{0}$. Premultiplying the first of the two conditions by $\mathbf{v}^{\dagger}$ and using $\mathbf{v}^{\dagger} \mathbf{u}=0$, we verify that the second requirement is automatically satisfied. It is sufficient to satisfy $\mathbf{g}_{m}(0)-$ $\mathbf{u v}^{\dagger} \mathbf{g}_{m}(1)=\mathbf{0}$. This proves the first part in (50). Next

$$
\begin{aligned}
\mathbf{G}_{m-2}^{-1}(z)= & \mathbf{G}_{m}^{-1}(z) \mathbf{V}_{2}(z) \\
= & \left(\mathbf{h}_{m}(0)+z \mathbf{h}_{m}(1)+\cdots\right) \\
& \times\left(\mathbf{u v} \mathbf{v}^{\dagger}+z^{-1} \mathbf{I}+s z^{-2} \mathbf{u v}\right)^{\dagger}
\end{aligned}
$$

The anticausality of this requires that the coefficients of $z^{-1}$ and $z^{-2}$ be zero. Proceeding as before, we obtain the second part in (50).

\section{REFERENCES}

[1] P. P. Vaidyanathan and T. Chen, "Role of anticausal inverses in multirate filter-banks-Part I: System-theoretic fundamentals," IEEE Trans. Signal Processing, this issue, pp. 1090-1102.

[2] F. R. Gantmacher, The Theory of Matrices. New York: Chelsea, 1959, vol. 1 and 2.

[3] P. P. Vaidyanathan, "Quadrature mirror filter banks, $M$-band extensions and perfect reconstruction techniques," IEEE Acoust. Speech Signal Processing Mag., vol. 4, pp. 4-20, July 1987.

[4] __, Multirate Systems and Filter Banks. Englewood Cliffs, NJ: Prentice Hall, 1993.

[5] P. P. Vaidyanathan, T. Q. Nguyen, Z. Doğanata, and T. Saramäki, "Improved technique for design of perfect reconstruction FIR QMF banks with lossless polyphase matrices," IEEE Trans. Acoustics, Speech, Signal Processing, vol. 37, pp. 1042-1056, July 1989.

[6] H. S. Malvar, "Lapped transforms for efficient transform/subband coding," IEEE Trans. Acoust. Speech Signal Processing, vol. 38, pp. 969-978, June 1990.

[7] P. Cassereau, "A new class of orthogonal transforms for image processing," S. M. thesis, Dept. EECS, Massachusetts Institute of Technology, Cambridge, MA, May 1985.

[8] H. S. Malvar, Signal Processing With Lapped Transforms. Norwood, MA: Artech House, 1992.

[9] A. V. Oppenheim and R. W. Schafer, Discrete-Time Signal Processing. Englewood Cliffs, NJ: Prentice Hall, 1989.

[10] G. H. Golub and C. F. Van Loan, Matrix Computations. Baltimore, MD: Johns Hopkins Univ. Press, 1989.

[11] R. A. Horn and C. R. Johnson, Matrix Analysis. Cambridge, MA: Cambridge Univ. Press, 1985.

[12] I. Daubechies, Ten Lectures on Wavelets, SIAM, CBMS series, Apr. 1992.

P. P. Vaidyanathan (S'80-M'83-SM'88-F'91), for a photograph and biography, please see page 1102 of this issue of this TRANSACTIONS.

Tsuhan Chen (S'90-M'93), for a photograph and biography, please see page 1102 of this issue of this Transactions. 